\title{
Cannon-Thurston maps for pared manifolds of bounded geometry
}

\author{
MAHAN MJ
}

\begin{abstract}
Let $N^{h} \in H(M, P)$ be a hyperbolic structure of bounded geometry on a pared manifold such that each component of $\partial_{0} M=\partial M-P$ is incompressible. We show that the limit set of $N^{h}$ is locally connected by constructing a natural Cannon-Thurston map. This provides a unified treatment, an alternate proof and a generalization of results due to Cannon and Thurston, Minsky, Bowditch, Klarreich and the author.
\end{abstract}

$57 \mathrm{M} 50$

\section{Introduction}

This is one in a series of papers (in between [36] and [39]) leading to the existence of Cannon-Thurston maps for, and local connectivity of limit sets of, finitely generated Kleinian groups. The project is completed in [38] and [40]. The main aim of this paper is to develop a reduction technique. Given the existence of Cannon-Thurston maps for closed surface groups of bounded geometry (cf [36]), this paper develops techniques to generalize this first to punctured surfaces and then to bounded geometry pared manifolds with incompressible boundary. While this is not a reduction theorem per se, the techniques of this paper shall be used mutatis mutandis in [39] and [40] to obtain generalizations of Cannon-Thurston theorems for surface groups to theorems for pared manifolds with incompressible boundary. The main focus of $[38 ; 39]$ and [40] will be to describe geometries of closed surface groups for which one can prove the existence of Cannon-Thurston maps. The main theorem of this paper is as follows.

Theorem (Theorem 5.12) Suppose that $N^{h} \in H(M, P)$ is a hyperbolic structure of bounded geometry on a pared manifold $(M, P)$ with incompressible boundary $\partial_{0} M=(\partial M-P)$ (ie each component of $\partial_{0} M$ is incompressible). Let $M_{\mathrm{gf}}$ denote a geometrically finite hyperbolic structure adapted to $(M, P)$. Then the map $i: \widetilde{M_{\mathrm{gf}}} \rightarrow$ $\widetilde{N^{h}}$ extends continuously to the boundary $\hat{i}: \widehat{M_{\mathrm{gf}}} \rightarrow \widehat{N^{h}}$. If $\Lambda$ denotes the limit set of $\widetilde{M}$, then $\Lambda$ is locally connected.

Examples to which Theorem 5.12 applies include the following. 
(1) The cover corresponding to the fiber subgroup of a closed hyperbolic 3-manifold fibering over the circle (Cannon and Thurston [15]),

(2) Hyperbolic 3 manifolds of bounded geometry, which correspond to simply or doubly degenerate Kleinian groups isomorphic to closed surface groups (Minsky [28]).

(3) Hyperbolic 3 manifolds of bounded geometry without parabolics and freely indecomposable fundamental group (Mitra [36, Section 4.3] and Klarreich [23]).

(4) Hyperbolic 3 manifolds of bounded geometry, arising from simply or doubly degenerate Kleinian groups corresponding to punctured surface groups (Bowditch $[11 ; 12])$.

The main issue addressed in this paper has been raised in various forms by Minsky [32], McMullen [26] and the author [33; 3].

Let $M$ be a closed hyperbolic 3-manifold fibering over the circle with fiber $F$. Let $\widetilde{F}$ and $\widetilde{M}$ denote the universal covers of $F$ and $M$ respectively. Then $\widetilde{F}$ and $\widetilde{M}$ are quasi-isometric to $\mathbb{H}^{2}$ and $\mathbb{H}^{3}$ respectively. Now let $\mathbb{D}^{2}=\mathbb{H}^{2} \cup \mathbb{S}_{\infty}^{1}$ and $\mathbb{D}^{3}=\mathbb{H}^{3} \cup \mathbb{S}_{\infty}^{2}$ denote the standard compactifications. In [15] Cannon and Thurston show that the usual inclusion of $\widetilde{F}$ into $\widetilde{M}$ extends to a continuous map from $\mathbb{D}^{2}$ to $\mathbb{D}^{3}$.

This was generalized by Minsky [28] and Klarreich [23] and independently (and using different techniques) by the author [36, Section 4] to prove that if $M$ is a geometrically tame hyperbolic 3-manifold with freely indecomposable fundamental group and injectivity radius bounded below, and if $M_{\mathrm{cc}}$ denotes the (topological) compact core of $M$ then the inclusion of $\widetilde{M}_{\text {cc }}$ into $\widetilde{M}$ extends to a continuous map from $\widehat{M}_{\mathrm{cc}}$ to $\widehat{M}$ where $\widetilde{M}_{\mathrm{cc}}$ and $\widetilde{M}$ denote the universal covers of $M_{\mathrm{cc}}$ and $M$ respectively and $\widehat{M}_{\mathrm{cc}}$ and $\widehat{M}$ denote the (Gromov) compactifications of $\widetilde{M}_{\mathrm{cc}}$ and $\widetilde{M}$ respectively. However, all these results left unanswered the case of manifolds with parabolics.

In [26] McMullen proved that the corresponding result holds for punctured torus groups, using the model manifold built by Minsky for these groups in [29]. In [39], we shall unify the framework of this paper with a certain notion of $i$-bounded geometry to give a simultaneous generalization of the results of this paper and those of McMullen [26].

The present paper was born of an attempt to find a new proof (along the lines of [36]) of a result of Bowditch. In $[11 ; 12]$, Bowditch proved the existence of Cannon-Thurston maps for punctured surface groups of bounded geometry using some of the ideas from the first paper in the present series by the author [36]. The main result of this paper 
simultaneously generalizes Bowditch's results $[11 ; 12]$ and $[36]$ and hence includes the results of $[15 ; 28 ; 23 ; 12 ; 11 ; 36]$. When $(M, P)$ is the pair $S \times I, \delta S \times I$, for $S$ a surface group, we get Bowditch's result $[11 ; 12]$. However, even in this case, our proof is different and circumvents the use of locally infinite (Farey) graphs as in the proofs of $[11 ; 12]$.

We are grateful to Brian Bowditch for pointing out that local connectivity (the second statement in Theorem 5.12 above) also follows by combining Bowditch's result [12] with a result of Anderson-Maskit [2]. See [12, Section 10] for details. The first part of Theorem 5.12 answers a question attributed to Thurston by Bowditch in the same paper [12, Section 10] (see also [1]).

In a sense, Theorem 5.12 above is a direct generalization of the following which we proved in [36].

Theorem (Theorem 4.7 of [36]) Let $M$ be a compact 3-manifold with incompressible boundary $\partial M$ without torus components. Let $M_{\text {hyp }}$ be a hyperbolic structure without parabolics on $M$ such that each end of the manifold has bounded geometry. Also suppose that $M_{\mathrm{gf}}$ is a geometrically finite structure on $M$. Then

- the inclusion of $\widetilde{M}_{\mathrm{gf}}$ into $\widetilde{M}_{\mathrm{hyp}}$ extends to a continuous map from $\widehat{M}_{\mathrm{gf}}$ to $\widehat{M}_{\text {hyp }}$

- the limit set of $\widetilde{M}_{\text {hyp }}$ is locally connected.

This problem is a part of a more general problem. A natural question seems to be the following.

Question $[36 ; 33 ; 3]$ Suppose $H$ is a hyperbolic group acting freely and properly discontinuously by isometries on a hyperbolic metric space $X$. Does the continuous proper map $i: \Gamma_{H} \rightarrow X$ extend to a continuous map $\widehat{i}: \widehat{\Gamma_{H}} \rightarrow \widehat{X}$ ?

We can ask the same question for relatively hyperbolic groups (in the sense of Gromov [20], Farb [17] and Bowditch [8]). A convenient framework for formulating this question is that of convergence groups acting on compacta (Bowditch [10]). Hyperbolic and relatively hyperbolic groups have been characterized in this setting by Bowditch [9] and Yaman [49].

Question Suppose a relatively hyperbolic group $H$ acts on a compact set $K$ such that the action is a convergence action and such that each 'cusp group' of $H$ leaves a point of $K$ fixed. Does there exist an equivariant continuous map from $\partial H$ to $K$ ? (Here $\partial H$ denotes the boundary of the relatively group $H$, which is well-defined as per [8].) 
A closely related question was raised by McMullen [26] in the context of Kleinian groups. However, his question deals with the Floyd completion rather than the Gromov completion as we have done here. There is some difference between the two notions for manifolds with cusps. Nevertheless, modulo Floyd's result [18], the two questions are equivalent in the context of Kleinian groups. This question has also gained attention after the recent resolution of the Ending Lamination Conjecture by Minsky [31] and Brock-Canary-Minsky [14]. In fact the question in the context of Kleinian groups has also been raised by Minsky in [32]. Both McMullen and Minsky ask if the limit sets of Kleinian groups are locally connected given that they are connected. This will be completely answered in the affirmative in [40].

Perhaps the most general context in which the question of local connectivity makes sense is that of convergence group actions on compacta [10].

Question Suppose a finitely generated group $H$ acts on a compact connected metrizable perfect set $K$ such that the action is a convergence action. Is $K$ locally connected? (ie does 'admitting a convergence action' promote a connected compactum to a Peano continuum?)

\subsection{Punctured surface groups of bounded geometry: A new proof}

As a motivational example, we sketch the proof for Kleinian groups $G$ that correspond to punctured surface groups, such that the 3-manifold $N^{h}=\mathbb{H}^{3} / G$ has bounded geometry away from the cusps, ie closed geodesics have length uniformly bounded away from zero. These are precisely the examples handled by Bowditch in [12] and [11]. We give a sketch in this particular case because these are the simplest new non-trivial examples and also to underscore the difference in our approach.

We first excise the cusps (if any) of $N^{h}$ leaving us a manifold that has one or two ends. Let $N$ denote $N^{h}$ minus cusps. Then $N$ is quasi-isometric to the universal curve over a Lipschitz path in Teichmuller space from which cusps have been removed. (In fact, in [36] we had proven that the path in question is a Teichmuller geodesic. The proof there was for closed surfaces, but can be extended painlessly to that of surfaces with punctures.) This path is semi-infinite or bi-infinite according as $N$ is one-ended or two-ended. Fix a reference finite volume hyperbolic surface $S^{h}$. Let $S$ denote $S^{h}$ minus cusps. Then $\widetilde{S}$ is quasi-isometric to the Cayley graph of $\pi_{1}(S)$ which is (Gromov) hyperbolic. We fix a base surface in $N$ and identify it with $S$. Now look at $\widetilde{S} \subset \widetilde{N}$. Let $\lambda=[a, b]$ be a geodesic segment in $\widetilde{S}$. We 'flow' $\lambda$ out the end(s) of $\widetilde{N}$ to generate a hyperbolic ladder-like set $B_{\lambda}$ (thinking of $\widetilde{S}$ as a horizontal sheet, see Figure 4) that looks topologically like $[a, b] \times[0, \infty)$ or $[a, b] \times(-\infty, \infty)$ according 
as $N$ has one or two ends. This is exactly a reproduction of our construction in [36] or [35].

A few details are in order. Regarding $N$ as a universal curve (minus cusps) over a Lipschitz path in Teichmuller space, we can assume that each fibre over the path is topologically $S$ with hyperbolic structure varying over the path. The union of all points that correspond to $a$ (or $b$ ) is a quasigeodesic ray (or bi-infinite quasigeodesic) in $\widetilde{N}$. Join the pairs of points that lie in the lift of a single copy of $\widetilde{S} \subset \widetilde{N}$, giving a geodesic in each copy of $\widetilde{S}$. This is what is meant by 'flowing' $\lambda$ out the end(s) with the two quasigeodesic rays mentioned above as 'guides' (or boundary lines), generating $B_{\lambda}$. The main technical theorems [35, Theorem 3.7] or [36, Theorem 3.8] ensure that there is a retraction from $\widetilde{N}$ to $B_{\lambda}$ which does not increase distances much. From this it follows that $B_{\lambda}$ is quasi-isometrically embedded in $\widetilde{N}$. [A brief proof of this last assertion, viz qi-embeddedness follows from retract, was given in [35] but we had omitted it in [36]. We are grateful to Brian Bowditch for pointing out this gap in [12]. See Gromov [20, Section 7.3J] for related result and [30] for a detailed proof of the same.] Note that for this we do not need $\widetilde{N}$ to be hyperbolic (in fact $\widetilde{N}$ is hyperbolic only when $S^{h}$ has no cusps and $\widetilde{N^{h}}=\widetilde{N}$. This is what we had used to prove the existence of Cannon-Thurston maps in [35] and [36]).

Now if $\lambda$ lies outside a large ball about a fixed reference point $p$ in $\widetilde{S}$, then so does $B_{\lambda}$ in $\widetilde{N}$. Since $B_{\lambda}$ is qi-embedded in $\widetilde{N}$, there exists an ambient $\widetilde{N}$-quasigeodesic $\mu$ lying in a bounded neighborhood of $B_{\lambda}$ joining the end-points of $\lambda$. If $S^{h}$ had no cusps, we immediately conclude that for any geodesic segment $\lambda$ in $\widetilde{S^{h}}$ lying outside large balls around $p$, there is a quasigeodesic in $\widetilde{N^{h}}$ joining its endpoints and lying outside a large ball around $p$ in $\widetilde{N^{h}}$. This gives us a continuous extension of the inclusion of $\widetilde{S^{h}}$ into $\widetilde{N^{h}}$ to the boundary. At this stage we have recaptured the results of Cannon and Thurston [15] and Minsky [28].

However, when $S^{h}$ has cusps, $\widetilde{S^{h}}$ and $\widetilde{S}$ are different. So a little more work is necessary. Suppose as before that $\lambda_{0}$ is a geodesic in $\widetilde{S^{h}}$ lying outside a large ball around $p$. For ease of exposition we assume that the end-points of $\lambda_{0}$ lie outside cusps. Let $\lambda \subset \widetilde{S}$ be the geodesic in (the path-metric on) $\widetilde{S}$ joining the same pair of points. Then off horodisks, $\lambda_{0}$ and $\lambda$ track each other. Construct $B_{\lambda}$ as before, and let $\mu$ be an ambient $\widetilde{N}$-quasigeodesic lying in a bounded neighborhood of $B_{\lambda}$ joining the end-points of $\lambda$. Then off horoballs in $\widetilde{N^{h}}, \mu$ lies outside a large ball around $p$. Let $\mu_{0}$ be the hyperbolic geodesic joining the end points of $\mu$. Off horoballs, $\mu$ and $\mu_{0}$ track each other. Hence, off horoballs, $\mu_{0}$ lies outside large balls about $p$. The points at which $\mu_{0}$ intersects a particular horoball therefore lie outside large balls about $p$. But then the hyperbolic segment joining them must do the same. This shows that $\mu_{0}$ 
must itself lie outside large balls around $p$. As before we conclude that there exists a continuous extension of the inclusion of $\widetilde{S^{h}}$ into $\widetilde{N^{h}}$ to the boundary. At this stage we have recaptured the result of Bowditch for punctured surfaces [11; 12].

We note here that the main purpose of this paper is to continue with our parallel approach to handling problems like the existence of a Cannon-Thurston map, and local connectivity of limit sets, initiated in [35] and [36]. These papers and the current one circumvent much of the sophisticated machinery specific to 2- and 3 manifolds developed by Thurston et al. Thus, they provide a direct approach to the existence of a Cannon-Thurston map, (as also local connectivity), without having to deal with laminations and associated geometries. Of course, this means that we lose out on the explicit description that Cannon and Thurston [15] or Minsky [28] or Bowditch [12] provide in terms of laminations. McMullen [26] also uses the notion of foliations in his proof to derive explicit information about locations of closed geodesics. However, the large-scale or coarse nature of our approach makes it more general, and suitable for application to cases where the surface machinery of Thurston is not available, eg general hyperbolic or relatively hyperbolic spaces and groups in the sense of Gromov, and more specifically, free groups where the notion of laminations developed by Bestvina, Feighn and Handel is substantially different $[5 ; 6]$. However, once the Cannon-Thurston map is in place, some notions of laminations can be resurrected, as in [34]. Thus, in principle, a long term aim of the present approach would be to derive further parallels between 3 manifolds and surfaces on the one hand, and discrete groups and their subgroups on the other. The latter context being vastly more general, we have tried to avoid the techniques specific to 2 and 3 dimensions.

The main technical difficulty in the first part of this paper (up to Section 4) stems from the fact that a closed geodesic on a component of the boundary of the pared manifold $\partial_{0} M=(\partial M-P)$ may be homotopic to a curve on a component of $P$, ie it is an accidental parabolic in any hyperbolic structure on $(M, P)$. This results in a somewhat trying case-by-case analysis in Section 4 of this paper. The resulting notation becomes a bit elaborate at times and we pause at a few moments during the course of the paper to summarize notation and keep it clear. There are essentially 3 cases we need to handle.

- $Z$-cusps these are relatively easy to handle as the above sketch shows.

- $(Z+Z)$ cusps where no curve on any component of $\partial_{0} M$ is homotopic to a curve on the boundary torus corresponding to the $(Z+Z)$ cusp.

- $Z$ and $(Z+Z)$ cusps, where some curve(s) on component(s) of $\partial_{0} M$ is(are) homotopic to a particular curve on the boundary torus corresponding to the $(Z+Z)$ cusp or some multiple of the core curve of the $Z$-cusp. 
We shall have occasion to introduce a certain extra hypothesis of $p$-incompressibility, but we stick to the general case, ie we do not introduce the extra hypothesis of $p$ incompressibility (or absence of accidental parabolics on components of $\partial_{0} M$ ) till Section 5.2, because the construction of $B_{\lambda}$ goes through even in the absence of this simplifying assumption. Further, once the proof is completed for the $p$-incompressible case, we use it (towards the end of Section 5) to prove the general case.

\subsection{Outline of the paper}

A brief outline of the paper follows. Section 2 deals with preliminaries on (Gromov) hyperbolic spaces. We also recall a result from McMullen [26] which says roughly that hyperbolic geodesics and ambient geodesics (geodesics in spaces obtained by removing some horoballs) track each other off horoballs.

In Section 3, we recall Thurston's definition of pared manifolds [47; 48] and show that the universal cover of a hyperbolic 3-manifold $M$, whose compact core is a pared manifold, is quasi-isometric to a tree $T$ of hyperbolic metric spaces with possibly exceptional vertex, once the cusps of $M$ have been removed. Further, if $\alpha$ denotes the root vertex, we demand that $\alpha$ be the possibly exceptional vertex. Let $X_{\alpha}$ denote the corresponding vertex space. We shall choose a geometrically finite structure $M_{\mathrm{gf}}$ on $M$ and identify $X_{\alpha}$ with ( $\widetilde{M_{\mathrm{gf}}}$ minus $Z$-cusps). This converts $X_{\alpha}$ into a hyperbolic metric space, but we relax the requirement that the embeddings of the edge spaces into $X_{\alpha}$ be qi-embeddings (this is what makes $\alpha$ exceptional). All other vertex and edge spaces are hyperbolic and all other inclusions of edge spaces into vertex spaces are qi-embeddings.

In Section 4, we modify the construction of [36] to construct a quasi-isometrically embedded hyperbolic ladder-like set $B_{\lambda}$ (see Figure 4) corresponding to a geodesic $\lambda$. We show that $B_{\lambda}$ is qi-embedded in ( $\widetilde{M}-Z$-cusps). $Z$-cusps and $(Z+Z)$-cusps are treated differently. The construction of $B_{\lambda}$ does not require $p$-incompressibility of the boundary components, but only their incompressibility.

It is in Section 5, that we first restrict the scope to pared manifolds with p-incompressible boundary components. We show that if $\lambda$ lies outside a large ball about a fixed reference point $p$ in $\left(X_{\alpha} \cup\right.$ cusps) modulo horoballs then so does $B_{\lambda}$ in $\widetilde{M}$.

Finally, we use the tracking properties of ambient quasigeodesics vis a vis hyperbolic geodesics and assemble the proof of the main theorem in the case of $p$-incompressible boundary. We deduce from this that the limit sets of the corresponding Kleinian groups are locally connected. Once this case is proven, we use it to prove the result for pared manifolds of incompressible boundary, thus relaxing the assumption on 
p-incompressibility. The concluding Section 6 deals with examples to which our theorem applies, notably Brock's example [13]. We also indicate possible directions of generalization. It would be worth bearing in mind that most of the arguments of this paper are relevant to the considerably more general framework of relatively hyperbolic groups a la Gromov [20], Farb [17] and Bowditch [8].

Apologia In From Beowulf to Virginia Woolf: An astounding and wholly unauthorized history of English literature [42] Robert Manson Myers claims that the plays usually attributed to Shakespeare are not in fact written by him but by another person of the same name. Being fully aware of our literary deficiencies we frankly admit that the papers $[35 ; 36]$ and the present one are written by the same person under different names.

Acknowledgements I am grateful to Brian Bowditch for several helpful comments on a previous version of this paper. I would also like to thank the referee for carefully reading the manuscript and suggesting several corrections and improvements. Research partly supported by a UGC Major Research Project grant.

Dedication To S V on his birthday.

\section{Preliminaries}

We start off with some preliminaries about hyperbolic metric spaces in the sense of Gromov [20]. For details, see [16; 19]. Let $(X, d)$ be a hyperbolic metric space. The Gromov boundary of $X$, denoted by $\partial X$, is the collection of equivalence classes of geodesic rays $r:[0, \infty) \rightarrow \Gamma$ with $r(0)=x_{0}$ for some fixed $x_{0} \in X$, where rays $r_{1}$ and $r_{2}$ are equivalent if $\sup \left\{d\left(r_{1}(t), r_{2}(t)\right)\right\}<\infty$. Let $\widehat{X}=X \cup \partial X$ denote the natural compactification of $X$ topologized the usual way (cf [19, page 124]).

The Gromov inner product of elements $a$ and $b$ relative to $c$ is defined by

$$
(a, b)_{c}=1 / 2[d(a, c)+d(b, c)-d(a, b)] .
$$

Definition 2.1 A subset $Z$ of $X$ is said to be $k$-quasiconvex if any geodesic joining points of $Z$ lies in a $k$-neighborhood of $Z$. A subset $Z$ is quasiconvex if it is $k$-quasiconvex for some $k$.

For simply connected real hyperbolic manifolds this is equivalent to saying that the convex hull of the set $Z$ lies in a bounded neighborhood of $Z$. We shall have occasion to use this alternate characterization. 
Definition 2.2 A map $f$ from one metric space $\left(Y, d_{Y}\right)$ into another metric space $\left(Z, d_{Z}\right)$ is said to be a $(K, \epsilon)$-quasi-isometric embedding if

$$
\frac{1}{K}\left(d_{Y}\left(y_{1}, y_{2}\right)\right)-\epsilon \leq d_{Z}\left(f\left(y_{1}\right), f\left(y_{2}\right)\right) \leq K d_{Y}\left(y_{1}, y_{2}\right)+\epsilon .
$$

If $f$ is a quasi-isometric embedding, and every point of $Z$ lies at a uniformly bounded distance from some $f(y)$ then $f$ is said to be a quasi-isometry. A $(K, \epsilon)$-quasiisometric embedding that is a quasi-isometry will be called a $(K, \epsilon)$-quasi-isometry.

A $(K, \epsilon)$-quasigeodesic is a $(K, \epsilon)$-quasi-isometric embedding of a closed interval in $\mathbb{R}$. A $(K, 0)$-quasigeodesic will also be called a $K$-quasigeodesic.

Let $\left(X, d_{X}\right)$ be a hyperbolic metric space and $Y$ be a subspace that is hyperbolic with the inherited path metric $d_{Y}$. By adjoining the Gromov boundaries $\partial X$ and $\partial Y$ to $X$ and $Y$, one obtains their compactifications $\widehat{X}$ and $\widehat{Y}$ respectively.

Let $i: Y \rightarrow X$ denote inclusion.

Definition 2.3 Let $X$ and $Y$ be hyperbolic metric spaces and $i: Y \rightarrow X$ be an embedding. A Cannon-Thurston map $\hat{i}$ from $\widehat{Y}$ to $\widehat{X}$ is a continuous extension of $i$.

The following lemma [35, Lemma 2.1] says that a Cannon-Thurston map exists if for all $M>0$ and $y \in Y$, there exists $N>0$ such that if $\lambda$ lies outside an $N$ ball around $y$ in $Y$ then any geodesic in $X$ joining the end-points of $\lambda$ lies outside the $M$ ball around $i(y)$ in $X$. For convenience of use later on, we state this somewhat differently.

Lemma 2.4 A Cannon-Thurston map from $\widehat{Y}$ to $\widehat{X}$ exists if the following condition is satisfied. Given $y_{0} \in Y$, there exists a non-negative function $M(N)$, such that $M(N) \rightarrow \infty$ as $N \rightarrow \infty$ and for all geodesic segments $\lambda$ lying outside an $N$-ball around $y_{0} \in Y$ any geodesic segment in $X$ joining the end-points of $i(\lambda)$ lies outside the $M(N)$-ball around $i\left(y_{0}\right) \in X$.

The above result can be interpreted as saying that a Cannon-Thurston map exists if the space of geodesic segments in $Y$ embeds properly in the space of geodesic segments in $X$.

We shall also be requiring certain properties of hyperbolic spaces minus horoballs. These were studied by Farb [17] under the garb of 'electric geometry'. We combine Farb's results with a version that is a (slight variant of a) theorem due to McMullen [26, Theorem 8.1]. 
Definition 2.5 A path $\gamma: I \rightarrow Y$ to a path metric space $Y$ is an ambient $K$-quasigeodesic if we have

$$
L(\beta) \leq K L(A)+K
$$

for any subsegment $\beta=\gamma \|[a, b]$ and any path $A:[a, b] \rightarrow Y$ with the same endpoints.

The following definitions are adapted from [17].

Definition 2.6 Let $M$ be a convex hyperbolic manifold. Let $Y$ be the universal cover of $M$ minus cusps and $X=\widetilde{M} \cdot \gamma$ is said to be a $K$-quasigeodesic in $X$ without backtracking if

- $\gamma$ is a $K$-quasigeodesic in $X$

- $\gamma$ does not return to any horoball $\mathbf{H}$ after leaving it.

$\gamma$ is said to be an ambient $K$-quasigeodesic in $Y$ without backtracking if

- $\gamma$ is an ambient $K$-quasigeodesic in $Y$

- $\quad \gamma$ is obtained from a $K$-quasigeodesic without backtracking in $X$ by replacing each maximal subsegment with end-points on a horosphere by a quasigeodesic lying on the surface of the horosphere.

Note that in the above definition, we allow the behavior to be quite arbitrary on horospheres (since Euclidean quasigeodesics may be quite wild); however, we do not allow wild behavior off horoballs.

$B_{R}(Z)$ will denote the $R$-neighborhood of the set $Z$. Let $\mathcal{H}$ be a locally finite collection of horoballs in a convex subset $X$ of $\mathbb{H}^{n}$ (where the intersection of a horoball, which meets $\partial X$ in a point, with $X$ is called a horoball in $X$ ). The following theorem is due to McMullen [26].

Theorem 2.7 [26] Let $\gamma: I \rightarrow X \backslash \bigcup \mathcal{H}=\mathcal{Y}$ be an ambient $K$-quasigeodesic for a convex subset $X$ of $\mathbb{H}^{n}$ and let $\mathcal{H}$ denote a collection of horoballs. Let $\eta$ be the hyperbolic geodesic with the same endpoints as $\gamma$. Let $\mathcal{H}(\equiv)$ be the union of all the horoballs in $\mathcal{H}$ meeting $\eta$. Then $\eta \cup \mathcal{H}(\eta)$ is (uniformly) quasiconvex and $\gamma(I) \subset B_{R}(\eta \cup \mathcal{H}(\equiv))$, where $R$ depends only on $K$.

The above theorem is similar in flavor to certain theorems about relative hyperbolicity a la Gromov [20], Farb [17] and Bowditch [8]. We give below a related theorem that is derived from Farb's 'Bounded Horosphere Penetration' property. 
Let $\gamma_{1}=\overline{p q}$ be a hyperbolic $K$-quasigeodesic without backtracking starting from a horoball $\mathbf{H}_{\mathbf{1}}$ and ending within (or on) a different horoball $\mathbf{H}_{2}$. Let $\gamma=[a, b]$ be the hyperbolic geodesic minimizing distance between $\mathbf{H}_{\mathbf{1}}$ and $\mathbf{H}_{\mathbf{2}}$. Following [17] we put the zero metric on the horoballs that $\gamma$ penetrates. The resultant pseudo-metric is called the electric metric. Let $\widehat{\gamma}$ and $\widehat{\gamma_{1}}$ denote the paths represented by $\gamma$ and $\gamma_{1}$ respectively in this pseudometric. It is shown in [17] that $\gamma, \widehat{\gamma}$ and $\widehat{\gamma_{1}}$ have similar intersection patterns with horoballs, ie there exists $C_{0}$ such that the following hold.

(1) If only one of $\gamma$ and $\widehat{\gamma_{1}}$ penetrates a horoball $\mathbf{H}$, then it can do so for a distance $\leq C_{0}$.

(2) If both $\widehat{\gamma_{1}}$ and $\gamma$ enter (or leave) a horoball $\mathbf{H}$ then their entry (or exit) points are at a distance of at most $C_{0}$ from each other. [Here by 'entry' (resp 'exit') point of a quasigeodesic we mean a point at which the path switches from being in the complement of or 'outside' (resp in the interior of or 'inside') a closed horoball to being inside (resp. outside) it].

The point to observe here is that quasigeodesics without backtracking in our definition gives rise to quasigeodesics without backtracking in Farb's sense. Since this is true for arbitrary $\gamma_{1}$ we give below a slight strengthening of this fact. Further, by our construction of ambient quasigeodesics without backtracking, we might just as well consider ambient quasigeodesics without backtracking in place of quasigeodesics.

Theorem 2.8 [17] Given $C>0$, there exists $C_{0}$ such that if

(1) either two quasigeodesics without backtracking $\gamma_{1}, \gamma_{2}$ in $X$, or

(2) two ambient quasigeodesics without backtracking $\gamma_{1}, \gamma_{2}$ in $Y$ or

(3) $\gamma_{1}$ - an ambient quasigeodesic without backtracking in $Y$ and $\gamma_{2}-$ a quasigeodesic without backtracking in $X$,

start and end

(1) either on (or within) the same horoball, or

(2) a distance $C$ from each other

then they have similar intersection patterns with horoballs (except possibly the first and last ones), ie there exists $C_{0}$ such that

(1) if only $\gamma_{1}$ penetrates or travels along the boundary of a horoball $\mathbf{H}$, then it can do so for a distance $\leq C_{0}$,

(2) if both $\gamma_{1}$ and $\gamma_{2}$ enter (or leave) a horoball $\mathbf{H}$ then their entry (or exit) points are at a distance of at most $C_{0}$ from each other. 


\section{Trees of hyperbolic metric spaces and pared manifolds}

\subsection{Definitions}

We start with a notion closely related to one introduced in Bestvina-Feighn [4].

Definition 3.1 A tree $T$ of hyperbolic metric spaces satisfying the q(uasi) i(sometrically) embedded condition is a metric space $(X, d)$ admitting a map $P: X \rightarrow T$ onto a simplicial tree $T$, such that there exist $\delta, \epsilon$ and $K>0$ satisfying the following.

(1) For all vertices $v \in T, X_{v}=P^{-1}(v) \subset X$ with the induced path metric $d_{v}$ is a $\delta$-hyperbolic metric space. Further, the inclusions $i_{v}: X_{v} \rightarrow X$ are uniformly proper, ie for all $M>0$, there exists $N>0$ such that for all $v \in T$ and $x, y \in X_{v}$, $d\left(i_{v}(x), i_{v}(y)\right) \leq M$ implies $d_{v}(x, y) \leq N$.

(2) Let $e$ be an edge of $T$ with initial and final vertices $v_{1}$ and $v_{2}$ respectively. Let $X_{e}$ be the pre-image under $P$ of the mid-point of $e$. Then $X_{e}$ with the induced path metric is $\delta$-hyperbolic.

(3) There exist maps $f_{e}: X_{e} \times[0,1] \rightarrow X$, such that $\left.f_{e}\right|_{X_{e} \times(0,1)}$ is an isometry onto the pre-image of the interior of $e$ equipped with the path metric.

(4) $\left.f_{e}\right|_{X_{e} \times\{0\}}$ and $\left.f_{e}\right|_{X_{e} \times\{1\}}$ are $(K, \epsilon)$-quasi-isometric embeddings into $X_{v_{1}}$ and $X_{v_{2}}$ respectively. $\left.f_{e}\right|_{X_{e} \times\{0\}}$ and $\left.f_{e}\right|_{X_{e} \times\{1\}}$ will occasionally be referred to as $f_{v_{1}}$ and $f_{v_{2}}$ respectively.

$d_{v}$ and $d_{e}$ will denote path metrics on $X_{v}$ and $X_{e}$ respectively. $i_{v}, i_{e}$ will denote inclusion of $X_{v}, X_{e}$ respectively into $X$.

We need a version of the above definition adapted to 3 manifolds with cusps. For convenience of exposition, $T$ shall be assumed to be rooted, ie equipped with a base vertex $\alpha$.

Definition 3.2 A tree $T$ of hyperbolic metric spaces with possibly exceptional vertex satisfying the q(uasi) $\mathrm{i}$ (sometrically) embedded condition is a metric space $(X, d)$ admitting a map $P: X \rightarrow T$ onto a rooted simplicial tree $T$ with root $\alpha$, such that there exist $\delta, \epsilon$ and $K>0$ satisfying the following.

(1) For all vertices $v \in T, X_{v}=P^{-1}(v) \subset X$ with the induced path metric $d_{v}$ is a $\delta$-hyperbolic metric space. Further, the inclusions $i_{v}: X_{v} \rightarrow X$ are uniformly proper, ie for all $M>0, v \in T$ and $x, y \in X_{v}$, there exists $N>0$ such that $d\left(i_{v}(x), i_{v}(y)\right) \leq M$ implies $d_{v}(x, y) \leq N$. 
(2) Let $e$ be an edge of $T$ with initial and final vertices $v_{1}$ and $v_{2}$ respectively. Let $X_{e}$ be the pre-image under $P$ of the mid-point of $e$. Then $X_{e}$ with the induced path metric is $\delta$-hyperbolic.

(3) There exist maps $f_{e}: X_{e} \times[0,1] \rightarrow X$, such that $\left.f_{e}\right|_{X_{e} \times(0,1)}$ is an isometry onto the pre-image of the interior of $e$ equipped with the path metric.

(4) $\left.f_{e}\right|_{X_{e} \times\{0\}}$ and $\left.f_{e}\right|_{X_{e} \times\{1\}}$ are $(K, \epsilon)$-quasi-isometric embeddings into $X_{v_{1}}$ and $X_{v_{2}}$ respectively for all $v_{1}, v_{2} \neq \alpha$. When one of $v_{1}, v_{2}$ is $\alpha$, this restriction is relaxed for the corresponding inclusion of edge spaces. $\left.f_{e}\right|_{X_{e} \times\{0\}}$ and $\left.f_{e}\right|_{X_{e} \times\{1\}}$ will occasionally be referred to as $f_{v_{1}}$ and $f_{v_{2}}$ respectively.

We shall work in the framework of pared manifolds in the sense of Thurston [47; 48].

Definition 3.3 A pared manifold is a pair $(M, P)$, where $P \subset \delta M$ is a (possibly empty) 2-dimensional submanifold with boundary such that

(1) the fundamental group of each component of $P$ injects into the fundamental group of $M$,

(2) the fundamental group of each component of $P$ contains an abelian subgroup with finite index,

(3) any cylinder $C:\left(S^{1} \times I, \delta S^{1} \times I\right) \rightarrow(M, P)$ with $C_{*}: \pi_{1}\left(S^{1} \times I\right) \rightarrow \pi_{1}(M)$ injective is homotopic rel. boundary to $P$,

(4) $P$ contains every component of $\delta M$ which has an abelian subgroup of finite index.

The terminology is meant to suggest that certain parts of the skin of $M$ have been pared off to form parabolic cusps in hyperbolic structures for $M . H(M, P)$ will denote the set of hyperbolic structures on $(M, P)$. (Note that this means that the elements of $P$ and the elements of $P$ alone are taken to cusps.)

Definition A pared manifold $(M, P)$ is said to have incompressible boundary if each component of $\partial_{0} M=\partial M \backslash P$ is incompressible in $M$.

Further, $(M, P)$ is said to have $p$-incompressible boundary if

(1) it has incompressible boundary,

(2) if some curve $\sigma$ on a component of $\partial_{0} M$ is freely homotopic in $M$ to a curve $\alpha$ on a component of $P$, then $\sigma$ is homotopic to $\alpha$ in $\partial M$.

$P_{0}, P_{1}$ will denote the components of $P$ whose fundamental groups are virtually $Z,(Z+Z)$ respectively. The adjective 'virtually' shall sometimes be omitted and we shall refer to the components of $P_{0}$ (resp. $\left.P_{1}\right)$ as $Z$-cusps (resp. $(Z+Z)$-cusps). 


\subsection{3 manifold as a tree of spaces}

The convex core of a hyperbolic 3-manifold $N^{h}$ is the smallest convex submanifold $C\left(N^{h}\right) \subset N^{h}$ for which inclusion is a homotopy equivalence.

If an $\epsilon$ neighborhood of $C\left(N^{h}\right)$ has finite volume, $N^{h}$ is said to be geometrically finite.

There exists a compact 3-dimensional submanifold $M_{\mathrm{cc}} \subset N^{h}$, the compact core or Scott core [44] whose inclusion is a homotopy equivalence. $M_{\mathrm{cc}}$ can be thought of as $C\left(N^{h}\right)$ minus cusps for geometrically finite $N^{h}$. $N^{h}$ minus cusps will be denoted by $N$ and $C\left(N^{h}\right)$ minus cusps will be denoted by $C(N)$. The ends of $N$ are in one-to-one correspondence with the components of $\left(N-M_{\mathrm{cc}}\right)$ or, equivalently, the components of $\partial_{0} M$.

We say that an end of $N$ is geometrically finite if it has a neighborhood missing $C(N)$.

Note The notion of ends here is slightly non-standard, as we do not want to regard a cusp as an end.

An end $E$ of $N$ is simply degenerate if it has a neighborhood homeomorphic to $S_{0} \times \mathbb{R}$, where $S_{0}$ is the corresponding component of $\partial_{0} M$, and if there is a sequence of pleated surfaces (with cusps removed) homotopic in this neighborhood to the inclusion of $S_{0}$, and exiting every compact set. Let $S^{h}$ denote a hyperbolic surface of finite volume, from which $S_{0}$ is obtained by excising cusps. We shall refer to $E^{h}=S^{h} \times \mathbb{R}$ (respecting the parameterization of $E$ ) as an end of $N^{h}$. Note that we may think of $E^{h}$ as obtained from $E$ by adjoining "half" a $Z$-cusp.

$N$ is called geometrically tame if all of its ends are either geometrically finite or simply degenerate. Note that $N^{h}$ and the interior of $N$ are homeomorphic to the interior of $M$. For a more detailed discussion of pleated surfaces and geometrically tame ends, see Canary-Epstein-Green [46] or Minsky [27].

A hyperbolic structure on $(M, P)$ is a complete hyperbolic metric on the interior of $M$ which takes precisely the elements of $P$ to cusps. A manifold $N^{h}$ will be said to be adapted to a pared manifold $(M, P)$ if $N^{h}$ corresponds to such a hyperbolic structure on $(M, P)$.

Note Since the flaring ends of $N$ contribute nothing to our discussion, we shall (abusing notation somewhat) regarding $\mathbf{N}=\mathbf{C}(\mathbf{N})$ and refer to $N$ as a hyperbolic manifold, though it should really be called a convex submanifold (minus cusps) homotopy equivalent to the big manifold (minus cusps). 
A manifold $M$ has bounded geometry if on a complement of the cusps, the injectivity radius of the manifold is bounded below by some number $\epsilon$ greater than 0 . Equivalently, all closed geodesics have length greater than $\epsilon$.

We want to first show that the universal cover of $N^{h}$ minus $Z$-cusps is quasi-isometric to a tree of hyperbolic metric spaces with possibly exceptional vertex corresponding to the core.

Let $E^{h}$ be a simply degenerate end of $N^{h}$. Then $E^{h}$ is homeomorphic to $S^{h} \times[0, \infty)$ for some surface $S^{h}$ of negative Euler characteristic. Cutting off a neighborhood of the cusps of $S^{h}$ we get a surface with boundary denoted as $S$. Let $E$ denote $E^{h}$ minus a neighborhood of the $Z$-cusps. We assume that each $Z$-cusp has the standard form coming from a quotient of a horoball in $\mathbb{H}^{3}$ by $Z$. Also, we shall take our pleated surfaces to be such that the pair ( $S$, cusps) is mapped to the pair ( $E$, cusps) for each pleated $S^{h}$. We shall now show that each $\widetilde{E}$ is quasi-isometric to a ray of hyperbolic metric spaces satisfying the qi-embedded condition. In [36] we had shown this for manifolds without cusps. Each edge and vertex space will be a copy of $\widetilde{S}$ and the edge to vertex space inclusions shall be quasi-isometries. Note that each $\widetilde{S}$ can be thought of as a copy of $\mathbb{H}^{2}$ minus an equivariant family of horodisks.

Lemma 3.4 [46] There exists $D_{1}>0$ such that for all $x \in E$, there exists a pleated surface $g:\left(S^{h}, \sigma\right) \rightarrow E^{h}$ with $g(S) \cap B_{D_{1}}(x) \neq \varnothing$. Also $g$ maps (S, cusps) to ( $E$, cusps).

The following lemma follows easily from the fact that $\operatorname{inj}_{N}(x)>\epsilon_{0}$.

Lemma 3.5 $[7 ; 46]$ There exists $D_{2}>0$ such that if $g:\left(S^{h}, \sigma\right) \rightarrow N^{h}$ is a pleated surface, then the diameter of the image of $S$ is bounded, ie $\operatorname{dia}(g(S))<D_{2}$.

The following lemma due to Thurston [46, Theorems 9.2 and 9.6.1] and Minsky [27] follows from compactness of pleated surfaces.

Lemma 3.6 [27] Fix $S^{h}$ and $\epsilon>0$. Given $a>0$ there exists $b>0$ such that if $g:\left(S^{h}, \sigma\right) \rightarrow E^{h}$ and $h:\left(S^{h}, \rho\right) \rightarrow E^{h}$ are homotopic pleated surfaces which are isomorphisms on $\pi_{1}$ and $E^{h}$ is of bounded geometry, then

$$
d_{E}(g(S), h(S)) \leq a \Rightarrow d_{\text {Teich }}(\sigma, \rho) \leq b,
$$

where $d_{\text {Teich }}$ denotes Teichmuller distance. 
In Lemma 3.6 $d_{E}$ denotes the path-metric on $E$ inherited from $N^{h}$. More precisely, the complete hyperbolic metric on $N^{h}$ gives rise to a path-metric on $N^{h} . E \subset N \subset N^{h}$ inherits a path-metric $d_{E}$ given by

$$
d_{E}(x, y)=\inf \{l(\sigma): \sigma \text { is a path in } E \text { joining } x, y\} .
$$

In [27] a special case of Lemma 3.6 is proven for closed surfaces. However, the main ingredient, a theorem due to Thurston is stated and proven in [46, Theorems 9.2 and 9.6.1] - 'algebraic limit is geometric limit' for finite area surfaces. The arguments given by Minsky to prove the above lemma from Thurston's Theorems (Lemma 4.5, Corollary 4.6 and Lemma 4.7 of [27]) go through with very little change for surfaces of finite area. In [12], Bowditch gives an alternate approach to this using (quite general) Gromov-Hausdorff limit arguments.

Note that in the above lemma, pleated surfaces are not assumed to be embedded. This is because immersed pleated surfaces with a uniform lower bound on injectivity radius are uniformly quasi-isometric to the corresponding Riemann surfaces.

Construction of equispaced pleated surfaces exiting the end We next construct a sequence of equispaced pleated surfaces $S^{h}(i) \subset E^{h}$ exiting the end. Assume that $S^{h}(0), \ldots, S^{h}(n)$ have been constructed such that:

(1) $S(i)$, cusps is mapped to $E$, cusps,

(2) if $E(i)$ is the component of $E \backslash S(i)$ for which $E(i)$ is non-compact, then $S(i+1) \subset E(i)$,

(3) Hausdorff distance between $S(i)$ and $S(i+1)$ is bounded above by $3\left(D_{1}+D_{2}\right)$,

(4) $d_{E}(S(i), S(i+1)) \geq D_{1}+D_{2}$,

(5) from Lemma 3.6 and condition (2) above there exists $D_{3}$ depending on $D_{1}, D_{2}$ and $S$ such that $d_{\text {Teich }}(S(i), S(i+1)) \leq D_{3}$.

Next choose $x \in E(n)$, such that $d_{E}\left(x, S_{n}\right)=2\left(D_{1}+D_{2}\right)$. Then by Lemma 3.4, there exists a pleated surface $g:\left(S^{h}, \tau\right) \rightarrow E^{h}$ such that $d_{E}(x, g(S)) \leq D_{1}$. Let $S^{h}(n+1)=g\left(S^{h}\right)$. Then by the triangle inequality and Lemma 3.5, if $p \in S(n)$ and $q \in S(n+1)$,

$$
D_{1}+D_{2} \leq d_{E}(p, q) \leq 3\left(D_{1}+D_{2}\right) .
$$

This allows us to continue inductively. $S(i)$ corresponds to a point $x_{i}$ of Teich $(S)$. Joining the $x_{i}$ in order, one gets a Lipschitz path $\sigma$ in $\operatorname{Teich}(S)$. 
Definition 3.7 A sequence of pleated surfaces satisfying conditions (1-5) above will be called an equispaced sequence of pleated surfaces. The corresponding sequence of $S(i) \subset E$ will be called an equispaced sequence of truncated pleated surfaces.

Since all $S^{h}(i)$ have bounded geometry away from cusps, they all lie in the thick part of Teichmuller space. After quotienting by the mapping class group, their images lie in a compact subset of the moduli space. Hence, by acting on $S^{h}(i)$ by some uniformly quasi-conformal map $\psi_{i}$, we may assume that $\psi_{i}\left(S^{h}(i)\right)$ gives rise to a fixed point $S^{h}$ in moduli space after quotienting by the mapping class group. Then $\psi_{i-1} \circ \psi_{i}^{-1}:\left(S^{h}(i)\right) \rightarrow\left(S^{h}(i-1)\right)$ is $C$-quasiconformal for some fixed $C$. We may assume that $\psi_{i} \circ \psi_{i-1}^{-1}:(S(i-1)) \rightarrow(S(i))$ is a bijective map by excising cusps appropriately. Also, assume that all these excised surfaces correspond to a fixed $S$ obtained from $S^{h}$ by excising cusps.

Definition 3.8 The universal curve over $X \subset \operatorname{Teich}\left(S^{h}\right)$ is a fiber bundle over $X$ whose fiber over $x \in X$ is the Riemann surface corresponding to $x$. (Topologically this is $X \times S^{h}$.)

Assuming that the $Z$-cusps are invariant under Teichmuller maps, we may assume that there is an induced universal curve of truncated hyperbolic surfaces obtained by excising cusps.

Each $S(i)$ being compact (with or without boundary), $\widetilde{S(i)}$ is a hyperbolic metric space. We want to regard the universal cover $\widetilde{E}$ of $E$ as being quasi-isometric to a ray $T$ of hyperbolic metric spaces. To this end, we construct a quasi-isometric model of $\widetilde{E}$. Let the following hold.

(1) $T=[0, \infty)$.

(2) Vertex set $\mathcal{V}=\{n: n \in \mathbb{N} \cup\{0\}\}$.

(3) Edge set $\mathcal{E}=\{[n-1, n]: n \in \mathbb{N}\}$.

(4) $X_{n}=\Gamma=X_{[n-1, n]}$, where $\Gamma$ is a Cayley graph of $\pi_{1}(S)$ with some fixed generating set.

(5) There exists $K, \epsilon$ and a map $\eta_{n}$ such that $\eta_{n}: \Gamma \rightarrow \widetilde{S(n)}$ is a $(K, \epsilon)$ quasiisometry for all $i$. Let $\eta_{n}^{-1}$ denote its quasi-isometric inverse.

(6) The qi-embeddings of edge sets into vertex sets are given by:

- $\phi_{n}: X_{[n-1, n]} \rightarrow X_{n}$ is the identity map on $\Gamma$,

- $\phi_{n-1}: X_{[n-1, n]} \rightarrow X_{n-1}$ is the change of marking induced by sending $\psi_{n-1}^{-1}(S)$ to $\psi_{n}^{-1}(S)$. 
By Lemma 3.6 and the fact that $\sigma$ is a Lipschitz path in Teichmuller space, this tree of hyperbolic metric spaces satisfies the quasi-isometrically embedded condition. In fact, we get more. Each $S(i)$ corresponds, via $S^{h}(i)$ to a point $x_{i}$ of Teich $(S)$. Joining the $x_{i}$ in order, one gets the Lipschitz path $\sigma$ obtained above in $\operatorname{Teich}(S)$. Mapping the fiber over $x_{i}$ to an embedded incompressible surface lying in a (uniformly) bounded neighborhood of the corresponding pleated surface and extending over product regions (using a metric product structure), we get a homeomorphism between the model and $E$. Further, since the Teichmuller distance between $S^{h}(i)$ and $S^{h}(i+1)$ is uniformly bounded above, the metric product is uniformly bi-Lipschitz to the region trapped between them in $E$. Pasting these homeomorphisms together and lifting to the universal cover, we get the following lemma.

Lemma 3.9 If $E^{h}$ is a simply degenerate end of a hyperbolic 3 manifold $N^{h}$ with bounded geometry, then there is a sequence of equispaced pleated surfaces exiting $E^{h}$ and hence a sequence of truncated pleated surfaces exiting $\widetilde{E}$. Further, $\widetilde{E}$ is quasiisometric to a ray of hyperbolic metric spaces satisfying the qi-embedded condition.

This lemma allows us to pass between $E$ and its quasi-isometric model, the ray of hyperbolic metric spaces satisfying the qi-embedded condition.

$Z$-cusps in $N$ correspond to $Z$-cusps in the boundary components of $\partial_{0} M$. But this is not true for $(Z+Z)$-cusps. Recall that $P_{0}$ (resp. $\left.P_{1}\right)$ denotes the components of $P$ whose fundamental group is virtually $Z$ (resp. $(Z+Z))$. Let $N_{0}=(N \cup$ $(Z+Z)$ cusps). We shall now describe the universal cover $\widetilde{N_{0}}$ as a tree of hyperbolic metric spaces with possibly exceptional vertex. It is at this stage that we need to assume that $N^{h}$ is adapted to a pared manifold $(M, P)$ with incompressible boundary. Recall that the incompressibility of the boundary $\partial_{0} M$ of a pared manifold does not require that $\partial M$ be incompressible, but only that the components of $\partial_{0} M=\partial M \backslash P$ be incompressible.

We give $\left(M, P_{1}\right)$ a geometrically finite structure (with no extra parabolics as per definition of a hyperbolic structure adapted to $\left.\left(M, P_{1}\right)\right)$ and denote the convex core of this geometrically finite manifold as $M_{0}$. Note that $M_{0}$ has no $Z$-cusps corresponding to $P_{0}$ but continues to have $(Z+Z)$ cusps corresponding to $P_{1}$. Further, since all $Z$-cusps have been excised in $N_{0}$, all the $(Z+Z)$-cusps in $N_{0}$ are retained in $M_{0}$.

Let $E(1), E(2), \ldots, E(k)$ denote the simply degenerate ends of ( $N_{0}-$ cusps). $M_{0}$ is homeomorphic by a homeomorphism that is a quasi-isometry to (the closure of) $N_{0} \backslash \bigcup_{i} E(i)$. We identify $M_{0}$ with its image under this map and denote $M_{0} \cap E(i)=$ $F(i)$, where $F(i)$ is both an embedded surface in $E(i)$ cutting off the end, and 
an incompressible boundary component of the pared manifold $\left(M_{0}, P_{1}\right)$. Note that $F(i)$ need not be a truncated pleated surface; but we have constructed $\widetilde{E}$ as a ray of hyperbolic metric spaces, and hence we only need $\widetilde{F(i)}$ to be quasi-isometric to $\Gamma$, a Cayley graph of the fundamental group $\pi_{1}(S)$.

Remark That there exists such a geometrically finite hyperbolic manifold homeomorphic to $N$ is part of Thurston's monster theorem. See McMullen [24; 25] for a different proof of the fact. Also, the limit set of a geometrically finite manifold is locally connected (Anderson-Maskit [2]). This shall be of use later.

Summary of notation We summarize here the notation introduced so far.

- $(M, P)$ - pared manifold.

- $P_{0} \subset P$ - components of $P$ whose fundamental group is virtually $Z$.

- $P_{1} \subset P$ - components of $P$ whose fundamental group is virtually $(Z+Z)$.

- $N^{h} \in H(M, P)$. Since flaring ends of $N^{h}$ do not contribute anything to the discussion, we identify $N^{h}$ with its convex core $C\left(N^{h}\right)$.

- $N=\left(N^{h}-\right.$ cusps $)$.

- $N_{0}=N$ with $(Z+Z)$-cusps (corresponding to $\left.P_{1}\right)$ adjoined.

- $M_{0}$ - geometrically finite structure on $\left(M, P_{1}\right)$.

- $E(i)$ for $i=1 \ldots k$ denote the ends of $N$. (Alternately, $E(i)=\left(E^{h}(i)-\right.$ cusps)).

- $M_{0}$ is identified with its quasi-isometric image and so is thought of as a subset of $N_{0}$.

- $N_{0}=M_{0} \cup \bigcup_{i} E(i)$.

- $F(i)=M_{0} \cap E(i)$.

Lemma 3.10 $\widetilde{N_{0}}$ is quasi-isometric to a tree $(T)$ of hyperbolic metric spaces satisfying the qi-embedded condition with possibly exceptional vertex $\alpha$ corresponding to $\widetilde{M_{0}} \subset$ $\widetilde{N_{0}}$.

Proof Note that $\widetilde{M_{0}} \subset \widetilde{N_{0}}$ is the universal cover of the convex core of a geometrically finite manifold and hence is a hyperbolic metric space. Let $\widetilde{F(i)} \subset \widetilde{N_{0}}$ represent a lift of $F(i)$ to $\widetilde{N_{0}}$. Then, $\widetilde{F(i)}$, being quasi-isometric to the fundamental group of a compact surface (with or without boundary) is a word-hyperbolic metric space. If $\widetilde{E(i)}$ is a lift of $E(i)$ containing $\widetilde{F(i)}$ then from Lemma 3.9, $\widetilde{E(i)}$ is a ray of hyperbolic metric spaces satisfying the qi-embedded condition. Since there are only finitely many ends $E_{i}$, we can thus regard $X=\widetilde{N_{0}}$ as a tree $(T)$ of hyperbolic metric spaces such that the following hold. 
- $\alpha$ is the root of $T . X_{\alpha}=\widetilde{M_{0}}$ is a hyperbolic metric space.

- $T$ consists of a (finite or infinite) collection of rays emanating from $\alpha$.

- Each copy of $\widetilde{E(i)}$ in $\widetilde{N_{0}}$ is quasi-isometric to a ray $\left(T_{i}\right)$ of hyperbolic metric spaces satisfying the qi-embedded condition (Lemma 3.9).

- $N_{0}=M_{0} \cup \bigcup_{i} E(i)$.

- $F(i)=M_{0} \cap E(i)$.

- As of now no restrictions are imposed on the inclusion of each $\widetilde{F}_{i}$ into $\widetilde{M_{0}}$.

These are precisely the defining conditions of a tree of hyperbolic metric spaces with possibly exceptional vertex satisfying the qi-embedded condition.

Note $T$ has a root vertex $\alpha$ which is possibly exceptional. The rest of $T$ consists of a number of rays emanating from $\alpha$.

\subsection{A topological property of pared manifolds}

Before we enter into the construction of quasiconvex sets, we shall describe a basic topological property of pared manifolds.

Lemma 3.11 Let $(M, P)$ be a pared manifold with incompressible boundary. Then

- no annulus component of $P$ is freely homotopic to a curve on a torus component or on another annulus component of $P$,

- if two curves (which are not non-trivial powers of any other curves) on $\partial_{0} M=$ $\partial M \backslash P$ are freely homotopic to curves on the same torus component of $P$, then they are in fact freely homotopic to the same curve on a torus component of $P$ and hence to each other.

Proof Statement 1 Let $\Delta$ be a boundary torus. If possible, let $A$ be an annulus in the boundary of $M$ such that its core curve is freely homotopic to a curve $\sigma$ on $\Delta$. The complement of a small neighborhood of $\sigma$ in $\Delta$ is an annulus $A_{1}$. Connecting the boundary curves of $A_{1}$ to those of $A$ by the free homotopy we get an immersed annulus with boundary on the boundary of $M$, but not homotopic to $\partial M$. This proves that the core curve of $A$ cannot be homotopic to the core curve of an annular component of $P$ as this would contradict condition 3 of the definition of a pared manifold. If two core curves of annuli components of $P$ are homotopic, we get a new annulus interpolating between these curves, again contradicting Condition 3 of the definition of a pared manifold. This proves the first part of the lemma. 
Statement 2 Let $M_{\Delta}$ denote the cover of $M$ corresponding to $\pi_{1}(\Delta)$. Let $\sigma_{1}$ and $\sigma_{2}$ be the curves homotopic to curves $\alpha_{1}$ and $\alpha_{2}$ on $\Delta$. Let $B$ denote the annulus between $\sigma_{1}$ and $\alpha_{1}$, then the intersection number between $\alpha_{1}$ and $\alpha_{2}$ in $\Delta$ is the same as the intersection number between $\alpha_{2}$ and $B$ in $M_{\Delta}$ (see for instance [46] or [7]). Since $\alpha_{2}, \sigma_{2}$ are homotopic in $M$, this homotopy lifts to $M_{\Delta}$. Hence, the intersection number between $\alpha_{2}$ and $B$ in $M_{\Delta}$ is the same as the intersection number between $\sigma_{2}$ and $B$ in $M_{\Delta}$ (by homotopy invariance of intersection numbers). If $\sigma_{1}$ and $\sigma_{2}$ are on different components then this latter number is zero.

Else, they belong to the same component $K$ of $\partial_{0}(M) . \pi_{1}(K)$ is either free nonabelian or else $K$ is a closed surface of genus greater than 1 . Since the fundamental group of $K$ injects into that of $M$, and since elements represented by $\sigma_{1}, \sigma_{2}$ commute, therefore $\sigma_{1}$ and $\sigma_{2}$ must be powers of the same curve. But the hypothesis says that the curves $\sigma_{1}$ and $\sigma_{2}$ are not powers of any other curves. Hence the two denote the same curve.

In either case, $\sigma_{1}$ and $\sigma_{2}$ are freely homotopic to the same curve on $\Delta$ and hence to each other.

\section{Construction of qi-embedded sets}

From Lemma 3.10 we have that $\widetilde{N_{0}}$ is quasi-isometric to a tree $T$ of hyperbolic metric spaces satisfying the qi-embedded condition with possibly exceptional vertex $\alpha$ corresponding to $\widetilde{M_{0}} \subset \widetilde{N_{0}}$. In fact $T$ has a root vertex $\alpha$ and consists of a number of distinct rays emanating from $\alpha$. Let $X$ denote the tree of spaces, $X_{v}$ denote vertex space corresponding to vertex $v, X_{e}$ denote edge space corresponding to edge $e$.

For convenience of exposition, we shall sometimes need to modify $X, X_{v}, X_{e}$ by quasiisometric perturbations and regard them as graphs. Given a geodesically complete metric space $(Z, d)$ of bounded geometry, choose a maximal disjoint collection $\left\{B_{1}\left(z^{\prime}\right)\right\}$ of disjoint 1-balls. Then by maximality, for all $z \in Z$ there exist $z^{\prime}$ in the collection such that $d\left(z, z^{\prime}\right)<2$. Construct a graph $Z_{1}$ with vertex set $\left\{z^{\prime}\right\}$ and edge set consisting of distinct vertices $z_{a}, z_{b}$ such that $d\left(z_{a}, z_{b}\right) \leq 4$. Then $Z_{1}$ equipped with the pathmetric is quasi-isometric to $(Z, d)$ (see for instance [21]). Henceforth we shall move back and forth between descriptions of spaces as Riemannian manifolds and as graphs, assuming that there is a quasi-isometry between them. The quasi-isometry will usually be suppressed.

Let $v$ be a vertex of $T$. Let $v_{-} \neq \alpha$ be the penultimate vertex on the geodesic edge path from $\alpha$ to $v$. Let $e_{-}$denote the directed edge from $v_{-}$to $v$. Define a quasi-isometry 
$\phi_{v}: f_{e_{-}}\left(X_{e_{-}} \times\{0\}\right) \rightarrow f_{e_{-}}\left(X_{e_{-}} \times\{1\}\right)$ as follows. If $p \in f_{e_{-}}\left(X_{e_{-}} \times\{0\}\right) \subset X_{v_{-}}$, choose $x \in X_{e_{-}}$such that $p=f_{e_{-}}(x \times\{0\})$ and define

$$
\phi_{v}(p)=f_{e_{-}}(x \times\{1\}) .
$$

Note that in the above definition, $x$ is arbitrarily chosen from a set of bounded diameter.

Let $\mu$ be a geodesic in $X_{v_{-}}$, joining $a, b \in f_{e_{-}}\left(X_{e_{-}} \times\{0\}\right) . \Phi_{v}(\mu)$ will denote a geodesic in $X_{v}$ joining $\phi_{v}(a)$ and $\phi_{v}(b)$. For our purposes since all the edge and vertex spaces on a ray, (apart from $X_{\alpha}$ ) are identical, we might as well identify $f_{e_{-}}\left(X_{e_{-}} \times\{0\}\right) \subset X_{v_{-}}$with $X_{v_{-}}$itself. Similarly we may identify $f_{e_{-}}\left(X_{e_{-}} \times\{1\}\right) \subset X_{v}$ with $X_{v}$ itself. Thus, $\phi_{v}$ is regarded as a quasi-isometry from $X_{v_{-}}$to $X_{v}$ for $v, v_{-} \neq \alpha$. We define a corresponding map $\Phi_{v}$ from geodesics in $X_{v_{-}}$to geodesics in $X_{v}$ by taking a geodesic joining $a, b \in X_{v_{-}}$to one joining $\phi_{v}(a), \phi_{v}(b) \in X_{v}$. From Lemma 3.9 , there exist $k, \epsilon>0$ such that for all $v, \phi_{v}$ is a $(k, \epsilon)$-quasi-isometry.

In Section 4.1, we shall construct a hyperbolic ladder-like set $B_{\lambda}$ containing $\lambda$. In Section 4.2 , we shall construct a retract $\Pi_{\lambda}: \widetilde{N_{0}} \rightarrow B_{\lambda}$. In Section 4.3 , we shall prove that $\Pi_{\lambda}$ is a retract, ie it fixes $B_{\lambda}$ and does not stretch distances much. This will show that $B_{\lambda}$ is quasi-isometrically embedded in $\widetilde{N_{0}}$.

\subsection{Construction of the hyperbolic ladder-like set $B_{\lambda}$}

The quasi-isometrically embedded set $B_{\lambda}$ that we intend to construct will contain the images of a geodesic under such quasi-isometries. Suppose $F(i)$ cuts off the end $E(i)$ and $\mu \subset \widetilde{F(i)} \subset \widetilde{E(i)}$. Then denote the union of the images of $\mu$ under the quasi-isometries taking it to the different vertex spaces as $B(\mu)$. That is to say, if $v_{0}, v_{1}, \ldots$ denote the vertices of the ray exiting the end, then the union of $\mu, \Phi_{v_{1}}(\mu)$, $\Phi_{v_{2}} \circ \Phi_{v_{1}}(\mu), \Phi_{v_{3}} \circ \Phi_{v_{2}} \circ \Phi_{v_{1}}(\mu)$, etc is denoted by $B(\mu)$.

Recall that $M_{0}$ denotes a convex geometrically finite hyperbolic manifold with hyperbolic structure adapted to the pared manifold $\left(M, P_{1}\right)$, where $P_{1} \subset P$ denotes the set of $(Z+Z)$-cusps. Also $\widetilde{M_{0}}$ the universal cover of $M_{0}$ is identified with $\widetilde{N_{0}}-\bigcup_{i} E(i) . M_{\mathrm{cc}}$ will denote $M_{0}$ minus a neighborhood of the $(Z+Z)$-cusps. Note that $M_{\mathrm{cc}}$ can be thought of as the Scott core of the manifold. $M_{\mathrm{gf}}$ will denote a geometrically finite hyperbolic structure adapted to the pared manifold $(M, P)$. The difference between $M_{\mathrm{gf}}$ and $M_{0}$ is that while $M_{\mathrm{gf}}$ is adapted to the pared manifold $(M, P), M_{0}$ is adapted to the pair $\left(M, P_{1}\right)$. Thus $M_{\mathrm{gf}}$ has incompressible boundary as a pared manifold, but the same may not be true of $M_{0}$. One may also think of $M_{\mathrm{gf}}$ as $M_{0}$ with $Z$-cusps (corresponding to $P_{0}$ ) adjoined. 
Fix a set of neighborhoods of the cusps of $M_{0}$, which are sufficiently separated from each other. Recall that $M_{\mathrm{cc}}$ denotes $M_{0}$ minus these cusps. Then $\widetilde{M_{\mathrm{cc}}}=\widetilde{M_{0}} \backslash \cup \mathcal{H}$ where $\mathcal{H}$ denotes an equivariant system of horoballs corresponding to lifts of $(Z+Z)-$ cusps. Note that $\widetilde{M_{\mathrm{cc}}}$ is quasi-isometric to the Cayley graph of $\pi_{1}(M)$ as the quotient is compact $[19 ; 20]$.

Let $\lambda^{h}=[a, b]$ be a hyperbolic geodesic in $\widetilde{M_{\mathrm{gf}}}$. Let $\beta^{h}$ be a hyperbolic geodesic in $\widetilde{N^{h}}$ joining $a, b$. Here $\widetilde{M_{\mathrm{gf}}}$ is identified with its image in $\widetilde{N^{h}}$. We shall show that if $\lambda^{h}$ lies outside a large ball around a fixed reference point $p \in \widetilde{M_{\mathrm{gf}}}$ then so does $\beta^{h} \in \widetilde{N^{h}}$. Recall that $F(i)$ for $i=1, \ldots, k$ are components of $\partial_{0} M=\partial M-P$. We can identify each $F(i)$ with a boundary component of $M_{0}$ so that the inclusion of $F(i)$ into $M_{\mathrm{cc}}$ induces an injection at the level of the fundamental group. We identify each $F(i)$ with the first truncated pleated surface exiting the end $E(i)$.

\section{Summary of Notation:}

- $M_{0}$ - convex geometrically finite hyperbolic structure on $\left(M, P_{1}\right)$.

- $M_{\mathrm{gf}}-$ convex geometrically finite hyperbolic structure adapted to the pared manifold $(M, P)$.

- $M_{\mathrm{cc}}-M_{0}$ minus $(Z+Z)$-cusps.

- $N^{h} \in H(M, P)$ has bounded geometry and is geometrically tame.

- $N-\left(N^{h}-\right.$ cusps $)$.

- $N_{0}-N$ with $(Z+Z)$ cusps adjoined.

- $M_{\mathrm{gf}}$ is identified with its homeomorphic image in $N^{h}$ taking cusps to cusps.

- $M_{0}$ is identified with its homeomorphic image in $N^{0}$.

- $M_{\text {cc }}$ is identified with its homeomorphic image in $N$.

Construction of $B_{0}(\lambda)$ Given $\lambda^{h}$ we shall first construct an ambient quasigeodesic $\lambda$ in $\widetilde{M_{0}}$. Since $\lambda^{h}$ is a hyperbolic geodesic in $\widetilde{M_{\mathrm{gf}}}$ there are unique entry and exit points for each horoball that $\lambda^{h}$ meets and hence unique Euclidean geodesics joining them on the corresponding horosphere. Replacing the segments of $\lambda^{h}$ lying inside $Z$-horoballs by the corresponding Euclidean geodesics, we obtain an ambient quasigeodesic $\lambda$ in $\widetilde{M_{0}}$ by Theorem 2.7. See Figure 1.

Again, since $\lambda$ coincides with $\lambda^{h}$ outside horoballs corresponding to $Z$-cusps, there exist unique entry and exit points of $\lambda$ into horoballs corresponding to $(Z+Z)-$ cusps, and hence again, unique Euclidean geodesics joining them on the corresponding horosphere. Replacing the segments of $\lambda$ lying inside $(Z+Z)$-horoballs by the 


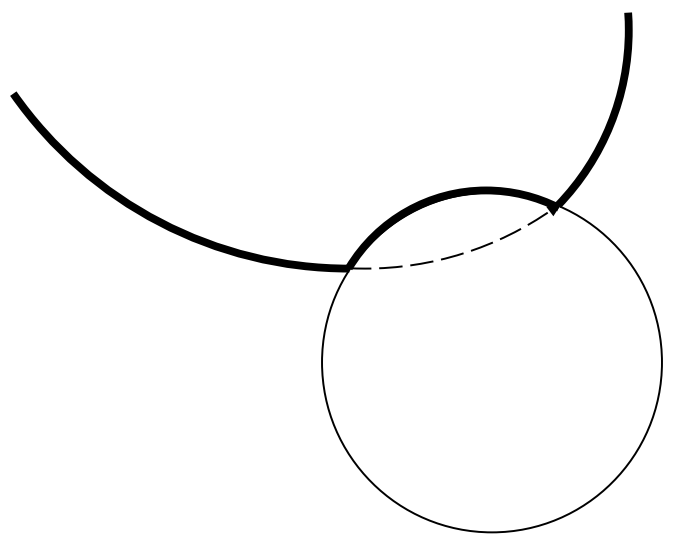

Figure 1

corresponding Euclidean geodesics, we obtain an ambient quasigeodesic $\lambda_{\mathrm{cc}}$ in $\widetilde{M_{\mathrm{cc}}}$ by Theorem 2.7. Each of the Euclidean geodesics mentioned above along with the hyperbolic geodesic joining its end-points (and lying entirely within the horoball) bounds a totally geodesic disk. (See Figure 2).

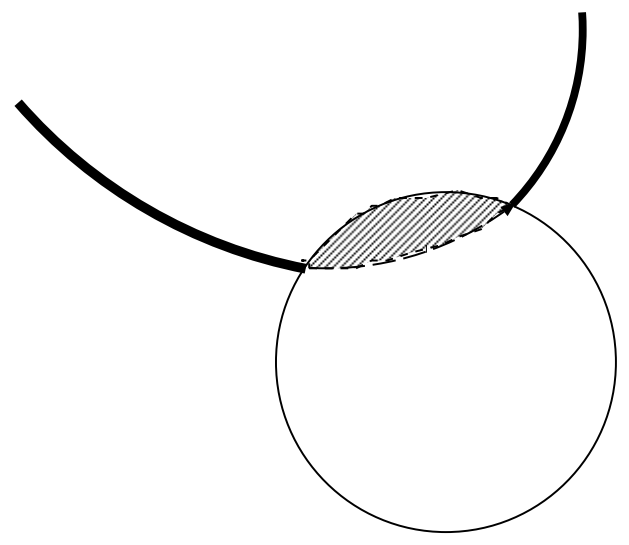

Figure 2 
The union of $\lambda$ and all these totally geodesic disks is denoted by $B_{0}(\lambda)$. There exists $C_{1}>0$ such that each $B_{0}(\lambda)$ is $C_{1}$-quasiconvex in $\widetilde{M_{0}}$. (See for instance McMullen [26, Section 8].)

$B_{0}^{\text {aug }}(\lambda)$ will denote $\lambda \cup \mathcal{H}(\lambda)$, where $\mathcal{H}(\lambda) \subset \mathcal{H}$ denotes the collection of horoballs in $\mathcal{H}$ that $\lambda$ meets. Again, from Theorem 2.7, $B_{0}^{\text {aug }}(\lambda)$ can be assumed to be $C_{1}-$ quasiconvex in $\widetilde{M_{0}}$.

Note We will be using $\widetilde{N_{0}}$ rather than $\widetilde{N^{h}}$ for the construction of $B_{\lambda}$. Hence $\widetilde{M_{0}}$ rather than $\widetilde{M_{\mathrm{gf}}}$ is the relevant space. Recall (Lemma 3.10) that $\widetilde{N_{0}}$ is a tree of hyperbolic metric spaces with possibly exceptional vertex corresponding to $\widetilde{M_{0}}$ satisfying the qi-embedded property.

Construction of $B_{1}(\lambda)$ Technically, (though not conceptually) this step is the most intricate one, and is the one new construction that is required to handle parabolics. The values of the constants chosen here become clear only through hindsight. The reason behind the choices made here will become clear only while constructing the projection in the next subsection. As mentioned in Section 1.1, we have to be quite careful while handling the different kinds of cusps that arise:

(1) Z-cusps,

(2) $(Z+Z)$ cusps, where no curve on any component of $\partial_{0} M$ is homotopic to a curve on the boundary torus corresponding to the $(Z+Z)$ cusp,

(3) $Z$ and $(Z+Z)$ cusps, where some curve(s) on component(s) of $\partial_{0} M$ is (are) homotopic to a particular curve on the boundary torus corresponding to the $(Z+Z)$ cusp or to some multiple of the core curve of a $Z$-cusp.

Next we need to consider the parts of $\lambda$ that can have substantial overlap with the ends $\widetilde{E}$, that is to say those pieces of $\lambda$ that follow some $\widetilde{F(i)}$ for a considerable length. There are geodesic segments on each $\widetilde{F(i)}$ parallel to these pieces. The union of all these parallel geodesic segments along with $B_{0}(\lambda)$ will be denoted by $B_{1}(\lambda)$. Details follow.

Lemma 4.1 Let $X$ be a convex subset of $\mathbb{H}^{n}$. Let $Y, Z$ be closed convex subsets of $X$. Let $Y_{\delta}=N_{\delta}(Y), Z_{\delta}=N_{\delta}(Z)$ be closed $\delta$-neighborhoods of $Y, Z$ respectively where $\delta$ denotes the hyperbolicity constant of the ambient space (in this case a convex subset of $\left.\mathcal{H}^{n}\right)$. Let $\Pi$ denote nearest point projection of $X$ onto $Y$. Then $d_{X}\left(\Pi(Z), Y_{\delta} \cap Z_{\delta}\right)$ is bounded in terms of $\delta$. 
Proof Case (a) $\left(Y_{\delta}\right) \cap\left(Z_{\delta}\right) \neq \varnothing$. Let $z \in Z$. We want to show that there exists $D=D(\delta)>0$ independent of $z$ such that $d_{X}\left(\Pi(z),\left(Y_{\delta}\right) \cap\left(Z_{\delta}\right)\right)<D$. Let $\Pi_{0}$ denote nearest point retraction of $X$ onto the convex set $\left(Y_{\delta}\right) \cap\left(Z_{\delta}\right)$ (which, being an intersection of convex sets, is convex).

As usual, $[a, b]$ denotes a geodesic joining $a, b$.

$$
[z, \Pi(z)] \subset N_{\delta}\left(\left[z, \Pi_{0}(z)\right] \cup\left[\Pi_{0}(z), \Pi(z)\right]\right) .
$$

But $\left[z, \Pi_{0}(z)\right] \subset Z_{\delta}$ and $\left[\Pi_{0}(z), \Pi(z)\right] \subset Y_{\delta}$. Hence,

$$
[z, \Pi(z)] \subset N_{\delta}(Y \cup Z) .
$$

- $z \in Z \subset Z_{\delta}$.

- $\Pi_{0}(z) \in Y_{\delta} \cap Z_{\delta}$.

- $\Pi(z) \in Y_{\delta}$.

The geodesic $[z, \Pi(z)]$ has to cross into $Y_{\delta}$ at some point $p \in Y_{\delta} \cap Z_{\delta}$. (Note that here we are using the fact that $Y_{\delta} \cap Z_{\delta}$ is closed.) But then $d(\Pi(z), p) \leq \delta$ (else we would be able to find another point $q$ at a distance of less than $\delta$ from $p$ in $Y$ contradicting the definition of $\Pi(z)$.) This proves Case (a).

Case (b) $\quad Y_{\delta} \cap Z_{\delta}=\varnothing$. Let $z_{1}, z_{2} \in Z$ and let $y_{i}=\Pi\left(z_{i}\right)$ for $i=1,2$. Since local quasi-geodesics are global quasi-geodesics $[20 ; 19]$ in hyperbolic metric spaces, there exists $D>0$ such that if $d\left(y_{1}, y_{2}\right) \geq D$, then $\left[z_{1}, y_{1}\right] \cup\left[y_{1}, y_{2}\right] \cup\left[y_{2}, z_{2}\right]$ is a $2 \delta$-quasigeodesic. (To see this, first note that [36, Lemma 3.1] ensures that $\left[z_{1}, y_{1}\right] \cup\left[y_{1}, y_{2}\right] \cup\left[y_{2}, z_{2}\right]$ is a $C=C(2 \delta)$-quasigeodesic. That $C=2 \delta$ comes from applying $\delta$-thinness to triangles $\left(z_{1}, y_{1}, y_{2}\right)$ and $\left(y_{1}, y_{2}, z_{2}\right)$ in succession.) In this case, $\left(\left[z_{1}, y_{1}\right] \cup\left[y_{1}, y_{2}\right] \cup\left[y_{2}, z_{2}\right]\right) \subset N_{2 \delta}\left[z_{1}, z_{2}\right]$. But $\left[z_{1}, z_{2}\right] \subset Z$ as $Z$ is convex. In particular $y_{1}, y_{2} \in N_{2 \delta}(Z)$. This contradicts the assumption that $Y_{\delta} \cap Z_{\delta}=\varnothing$. Hence, $d\left(y_{1}, y_{2}\right) \leq D$, proving the lemma.

The above lemma can be 'quasi-fied' as follows. (The scheme of proof being a 'quasification' of the above, we omit it.)

Lemma 4.2 Given $\delta, C>0$, there exists $k>0$ such that the following holds. Let $X$ be a $\delta$-hyperbolic metric space and $Y, Z$ be $C$-quasiconvex subsets of $X$. Let $Y_{k}, Z_{k}$ denote $k$-neighborhoods of $Y, Z$ respectively. Let $\Pi$ denote a nearest point projection of $X$ onto $Y$. Then $d_{X}\left(\Pi(Z),\left(Y_{k} \cap Z_{k}\right)\right)$ is uniformly bounded. Hence there exists $k>0$ such that $\left(\Pi(Z) \subset\left(Y_{k} \cap Z_{k}\right)\right)$ if the latter set is non-empty and $\Pi(Z)$ has diameter bounded by $k>0$ if $Y_{k} \cap Z_{k}=\varnothing$. 
Let $F$ be one of the $F(i)$. Either $\widetilde{F}$ is quasiconvex in $\widetilde{M_{0}}$ (when there are no accidental parabolics) or there exist disjoint curves $\sigma_{i}$ for $i=1, \ldots, l$ (by Lemma 3.11) homotopic to curves on components of $P$. Therefore $\sigma_{i}$ correspond to parabolics. Each $\sigma_{i}$ lies at a bounded distance from some Euclidean geodesic $\eta_{i}$ on a torus or annular component of $P$ in $M_{\mathrm{cc}}$. Each $\eta_{i}$ forms the boundary of a totally geodesic $Z$ cusp $\kappa_{i}$ (possibly a totally geodesic subset of a $Z+Z$-cusp). Further, the two curves $\eta_{i}$ and $\sigma_{i}$ bound between themselves an annulus $A_{i}$. Let $G=F \cup \bigcup_{i}\left(A_{i} \cup \kappa_{i}\right)$. Then $\widetilde{G}$ is quasiconvex in $\widetilde{M_{0}}$. Choose a constant $C_{2}>0$ such that each such $\widetilde{G}$ is $C_{2}$-quasiconvex in $\widetilde{M_{0}}$. Recall that $B_{0}(\lambda)$ and $B_{0}^{\text {aug }}(\lambda)$ are $C_{1}$-quasiconvex.

We are now in a position to start constructing $B_{1}(\lambda)$ from $B_{0}(\lambda)$.

Choosing $Y=B_{0}(\lambda)$ and $Z=\widetilde{G(i)}$ (one lift of $G(i)$ is considered in turn, and choosing $k$ to be the maximum of the corresponding finite collection of $k$ ), we obtain a $k>0$ from Lemma 4.2 above, so that $\left(\Pi(Z) \subset\left(Y_{k} \cap Z_{k}\right)\right)$. Let $\widetilde{F(i)}$, $i=1, \ldots, s$ denote the different lifts of the $F_{i}$ that intersect a $(3 k+4 \delta)$ neighborhood of $B_{0}(\lambda)$. Since $\lambda$ is a finite segment, the number $s$ is finite. Choose $p_{i}, q_{i} \in$ $\widetilde{F(i)} \cap N_{3 k+4 \delta}\left(B_{0}(\lambda)\right)$ such that $d\left(p_{i}, q_{i}\right)$ is maximal.

Fix $D>0$. Choose the copies of $\widetilde{F(i)}$ for which $d\left(p_{i}, q_{i}\right) \geq D$.

Note The number $D$ picks up significance in the $p$-incompressible case. We shall come back to this in Section 5.2, when we simplify our problem under this extra assumption.

Redefining $s$ if necessary, we let $\widetilde{F(1)}, \ldots, \widetilde{F(s)}$ denote this collection. Let $\mu_{i}$ denote the geodesic in $\widetilde{F(i)}$ joining $p_{i}$ and $q_{i}$. Let $\mathcal{E}$ denote the corresponding collection of $\widetilde{E(i)}$. Let $\mathcal{E}^{\prime}$ denote the collection of the remaining $\widetilde{E(i)}$. We would like to define

$$
B_{1}(\lambda)=B_{0}(\lambda) \cup \bigcup \mu_{i}
$$

The choice of $\mathcal{E}, \mathcal{E}^{\prime}$ is dependent on $k$. We want each $\widetilde{E}$ to be such that

- either $\widetilde{F}$ is quasiconvex in $\widetilde{M_{0}}$ (case where no curve on $F$ is parabolic in $\widetilde{M_{0}}$ ),

- or, $\widetilde{G}$ (which is always quasiconvex in $\widetilde{M_{0}}$ ) intersects at most one $\mathbf{H} \in \mathcal{H}\left({ }^{(}\right)$.

Lemma 4.3 There exists $k^{\prime}>0$ such that if $\widetilde{G}$ intersects more than one horoball in $\mathcal{H}(\lambda)$, then $N_{k^{\prime}}\left(B_{0}(\lambda)\right) \cap \widetilde{F}$ has non-zero diameter. 
Proof Suppose $\widetilde{G}$ intersects more than one horoball $\mathbf{H} \in \mathcal{H}\left(\widetilde{)}\right.$, say $\mathbf{H}_{1}, \mathbf{H}_{2}$. Let $p \in \mathbf{H}_{1} \cap \widetilde{G}$ and $q \in \mathbf{H}_{2} \cap \widetilde{G}$. Let $[p, q]_{0}$ denote the geodesic in $\widetilde{G}$ joining $p, q$, and let $[p, q]$ denote the geodesic in $\widetilde{M_{0}}$ joining $p, q$. $[p, q]_{0}$ lies in a $C_{1}$-neighborhood of $[p, q]$ as $\widetilde{G}$ is $C_{1}$-quasiconvex. Since horoballs are sufficiently separated in both $\widetilde{G}$ and $\widetilde{M_{0}}$, therefore some part of $[p, q]_{0}$ lies on $\widetilde{F}$. Hence $N_{C_{1}}([p, q]) \cap \widetilde{F} \neq \varnothing$ and has non-zero diameter.

Next, there is a subsegment of $\lambda$ starting in $\mathbf{H}_{1}$ and ending in $\mathbf{H}_{2}$. Let $\lambda^{\prime}$ be the part of this subsegment lying outside the interior of the horoballs $\mathbf{H}_{1}$ and $\mathbf{H}_{2}$. Then, off horoballs, $\lambda^{\prime}$ and $[p, q]$ track each other (by Theorem 2.8), ie there exists $C_{2}>0$ such that $[p, q] \subset N_{C_{2}}(\lambda \cup \mathcal{H}(\lambda))$ and the subsegment of $[p, q]$ lying outside the horoballs $\mathbf{H}_{1}$ and $\mathbf{H}_{2}$ is contained in a $C_{2}$-neighborhood of $B_{0}(\lambda)$. Choosing $k^{\prime}=C_{1}+C_{2}$, we are through.

The next corollary follows.

Corollary 4.4 Given $k>0$, there exists $k^{\prime}>0$ such that if the $2 k$-neighborhood of $\widetilde{G}$ intersects more than one horoball in $\mathcal{H}(\lambda)$, then the diameter of $N_{k^{\prime}}\left(B_{0}(\lambda)\right) \cap \widetilde{F}$ is non-zero.

Here the term $2 k$ occurs so as to recall the $k$ of Lemma 4.2. Choose $K=3 k+4 \delta+k^{\prime}$, where $k$ is as in Lemma 4.2 and $k^{\prime}$ is as in Corollary 4.4 above.

We now return to our construction of $B_{1}(\lambda)$, or more specifically the $\mu_{i}$ that we had mentioned after Lemma 4.2.

Let $\widetilde{F(i)}, i=1, \ldots, s$ (redefining $s$ if necessary) denote the different lifts of the $F(i)$ that intersect a $K$ neighborhood of $B_{0}(\lambda)$. Since $\lambda$ is a finite segment, the number $s$ is finite. Choose $p_{i}, q_{i} \in \widetilde{F(i)} \cap N_{3 k+4 \delta}\left(B_{0}(\lambda)\right)$ such that $d\left(p_{i}, q_{i}\right)$ is maximal.

Recall that $p_{i}, q_{i} \in \widetilde{F(i)} \cap N_{3 k+4 \delta}\left(B_{0}(\lambda)\right)$ for some copies of $\widetilde{F(i)}$. Choose the copies of $\widetilde{F(i)}$ for which $d\left(p_{i}, q_{i}\right)>0$. Redefining $s$ once more, if necessary, we let $\widetilde{F(1)}, \ldots, \widetilde{F(s)}$ denote this collection. Let $\mu_{i}$ denote the geodesic in $\widetilde{F(i)}$ joining $p_{i}$ and $q_{i}$. Let $\mathcal{E}$ denote the corresponding collection of $\widetilde{E(i)}$. Let $\mathcal{E}^{\prime}$ denote the collection of the remaining $\widetilde{E(i)}$. We are finally in a position to define

$$
B_{1}(\lambda)=B_{0}(\lambda) \cup \bigcup \mu_{i}
$$

See Figure 3 below. $\lambda$ lies on $\widetilde{M_{0}}$. 'Parallel' segments $\mu_{1}, \ldots, \mu_{k}$ are constructed lying on $\widetilde{F(1)}, \ldots, \widetilde{F(k)}$. 


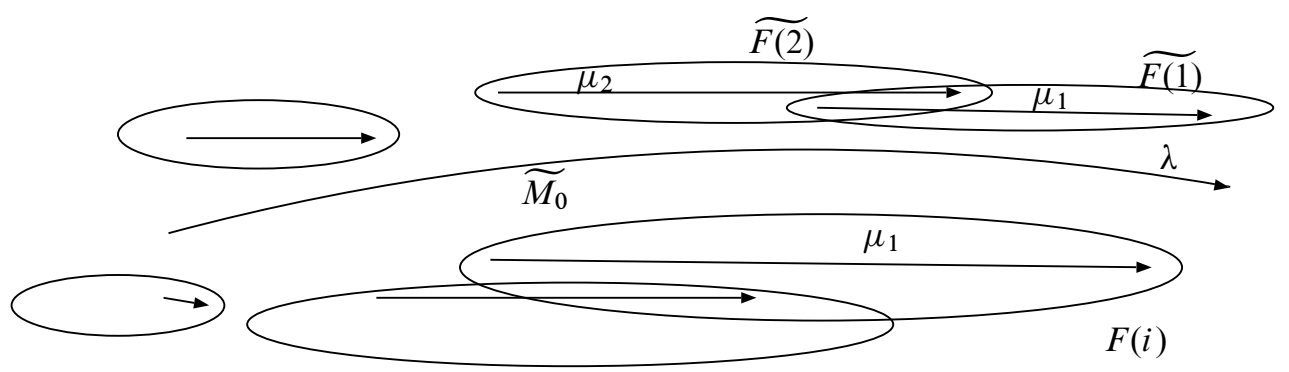

Figure 3

Construction of $B(\mu) \quad$ Recall from Lemma 3.9 that each $\widetilde{E}$ is quasi-isometric to a ray $[0, \infty)$ of hyperbolic metric spaces with integer points corresponding to vertices and $[n-1, n]$ (for $n \in \mathbb{N}$ ) corresponding to edges. Fix a particular $\widetilde{F}$ cutting off the end $\widetilde{E}$ and a geodesic segment $\mu \subset \widetilde{F}$. Let $X_{i}$ denote the vertex spaces for $i=0,1, \ldots$ Let $\phi_{i}$ denote the quasi-isometry from $X_{i-1}$ to $X_{i}$ and $\Phi_{i}$ denote the corresponding map from geodesic segments in $X_{i-1}$ to those in $X_{i}$. Define

$$
\mu^{i}=\Phi_{i} \circ \cdots \circ \Phi_{1}(\mu) \text { and } B(\mu)=\bigcup \mu^{i} .
$$

See Figure 4 below.

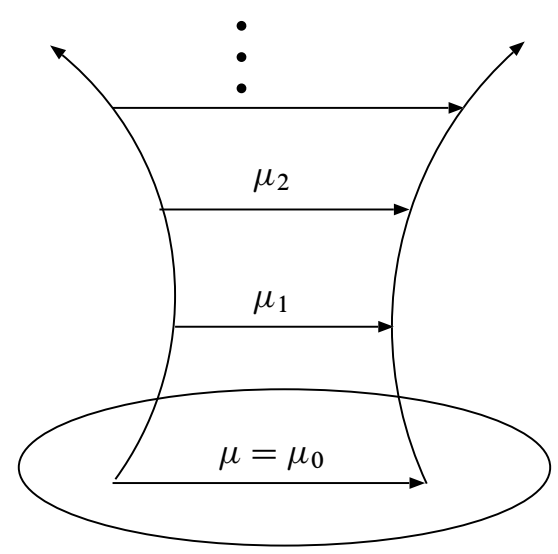

Figure 4: Hyperbolic ladder-like Set

Definition of $B_{\lambda} \quad$ Finally define

$$
B_{\lambda}=B_{1}(\lambda) \cup \bigcup_{i=1 \ldots s} B\left(\mu_{i}\right)
$$




\subsection{Definition of $\Pi_{\lambda}$}

Recall $\mathcal{E}=\{\widetilde{E(1)}, \ldots, \widetilde{E(s)}\}$ and $\mathcal{E}^{\prime}=\{\widetilde{E(s+1)}, \widetilde{E(s+2)}, \ldots\}$. We next want to show that $B_{\lambda}$ is quasi-isometrically embedded. To prove this, we shall construct a retraction $\Pi_{\lambda}$. Let $X_{i j}$ denote the vertex spaces in $\widetilde{E(i)} \in \mathcal{E}$. Below, we shall have need to replace $\widetilde{E(i)}$ by the union of vertex spaces $\bigcup_{j} X_{i j}$ (fixing $i$ and letting $j$ vary from 0 to $\infty$ ) with the understanding that the latter has the metric induced from $\widetilde{E(i)}$. The difference between $\widetilde{E(i)}$ and $\bigcup_{j} X_{i j}$ is just that the edge spaces of $\widetilde{E(i)}$ are not explicitly present in the latter.

Let $\phi_{i j}$ denote the quasi-isometry from $X_{i, j-1}$ to $X_{i j}$ and let $\Phi_{i j}$ denote the induced map from geodesics in $X_{i, j-1}$ to geodesics in $X_{i j}$. Let $\mu_{i j} \subset X_{i j}$ denote the image of $\mu_{i}$ under the composition of the maps $\Phi_{i k}$ as $k$ runs from 1 to $j$.

- Let $\Pi_{i j}$ denote a nearest point retraction onto $\mu_{i j} \subset X_{i j}$. On $\widetilde{E(i)}=\bigcup_{j} X_{i j}$, $\Pi_{i}$ is defined by

$$
\Pi_{i}(x)=\Pi_{i j}(x) \text { for } x \in X_{i j} .
$$

- On $\widetilde{M_{0}}=X_{\alpha}$ (recall that $\alpha$ corresponds to the possibly exceptional vertex of the tree of spaces) let $\Pi_{0}$ denote a nearest point retraction onto $B_{0}(\lambda)$.

On the remaining set $\mathcal{E}^{\prime}, \Pi_{\lambda}$ needs to be defined with some caution. Suppose $\widetilde{E} \in \mathcal{E}^{\prime}$. Observe first that no point on $\widetilde{F} \subset \widetilde{E}$ lies at a distance less than $K$ from $B_{0}(\lambda)$, where $K$ is as chosen after Corollary 4.4. Also let $k$ be as in Lemma 4.2.

Case (a) $\quad N_{2 k}(\widetilde{G})$ does not intersect $\mathcal{H}(\lambda)$. In this case, choose $y \in \widetilde{F}$ arbitrarily and define

$$
\Pi_{E}(x)=\Pi_{0}(y) \text { for all } x \in \widetilde{E} .
$$

For $\widetilde{E}=\widetilde{E(i)}$, denote $\Pi_{E}$ by $\Pi_{i}$.

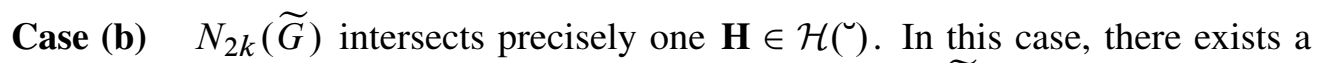
unique lift $\widetilde{\sigma}$ of a curve $\sigma$ on $F$, (parabolic in $M_{0}$ ) lying on $\widetilde{F}$ at a bounded distance from $\mathbf{H}$.

As in the construction of $B(\mu)$ for $\mu \subset \widetilde{F} \subset \widetilde{E}$, construct $B(\widetilde{\sigma})=\bigcup_{j} \widetilde{\sigma}_{j} \subset \bigcup_{j} \widetilde{S(j)}$ where $S(j)$ denotes the $j$ th member of a sequence of truncated pleated surfaces exiting $E$ and $\widetilde{\sigma}_{j}=\Phi_{j}\left(\widetilde{\sigma}_{j-1}\right)\left(\Phi_{j}\right.$ is the map on geodesics induced by the quasi-isometry $\phi_{j}$ from $\widetilde{S(j-1)}$ to $\widetilde{S(j))}$. Note that this construction works just as well for infinite geodesics. On $\widetilde{S(j)}$, define $\Pi_{\sigma j}$ to be a nearest point projection onto $\widetilde{\sigma}_{j}$. Define

$$
\Pi_{\sigma}^{0}(x)=\Pi_{\sigma j}(x) \text { for } x \in \widetilde{S(j)} .
$$


Next, let $\Pi_{\sigma}$ denote nearest point projection of $\widetilde{E}$ onto $\widetilde{\sigma}$. Define

$$
\Pi_{E}(x)=\Pi_{0} \circ \Pi_{\sigma}(x) \circ \Pi_{\sigma}^{0}(x) \text { for all } x \in \widetilde{E} .
$$

For $\widetilde{E}=\widetilde{E(i)}$, denote $\Pi_{E}$ by $\Pi_{i}$.

The reason for factoring the projection $\Pi_{\sigma}(x)$ through $\Pi_{\sigma}^{0}(x)$ is that on $\widetilde{F(i)}$ we want $\Pi_{i}$ to coincide with $\Pi_{\sigma}$. (Else nearest point projections will have to be taken in the $\widetilde{E(i)}$ metric and $\widetilde{F(i)}$ is not quasiconvex in this metric, so that nearest point projections might well differ substantially in the two metrics.)

Non-Definition We would like to define

$$
\begin{aligned}
\Pi_{\lambda}(x) & =\Pi_{i}(x), x \in \widetilde{E(i)} \in \mathcal{E} \\
& =\Pi_{0}(x), x \in X_{\alpha} \\
& =\Pi_{i}(x), x \in \widetilde{E(i)} \in \mathcal{E}^{\prime} .
\end{aligned}
$$

Caveat Our non-definition above is not yet a real definition as $\Pi_{\lambda}$ has been putatively defined twice on each $\widetilde{F(i)}$, once regarding $\widetilde{F(i)}$ as a subset of $\widetilde{M_{0}}$ and again, regarding it as a subset of $\widetilde{E(i)}$. We will show that there is at most a bounded amount of discrepancy between the two definitions and so any choice will work. So we make the following definition.

\section{Definition}

$$
\begin{aligned}
\Pi_{\lambda}(x) & =\Pi_{i}(x), x \in \widetilde{E(i)}-X_{i 0}, \widetilde{E(i)} \in \mathcal{E} \\
& =\Pi_{0}(x), x \in X_{\alpha} \\
& =\Pi_{i}(x), x \in \widetilde{E(i)} \in \mathcal{E}^{\prime} .
\end{aligned}
$$

\section{3 $\Pi_{\lambda}$ is a retract}

We will show in this subsection that there exists $C>0$ such that for all $x, y \in X=$ $\widetilde{N_{0}}, d\left(\Pi_{\lambda}(x), \Pi_{\lambda}(y)\right) \leq C d(x, y)$ There are three parts in the definition of $\Pi_{\lambda}$ as described above. We discuss the three cases separately.

Since we are dealing with graphs, it suffices to prove the result for $d(x, y)=1$. We need to check the following.

(1) $x \in \widetilde{F(i)}=\widetilde{E(i)} \cap \widetilde{M_{0}}$ for some $\widetilde{E(i)} \in \mathcal{E}$. We want to show that

$$
d\left(\Pi_{i}(x), \Pi_{0}(x)\right) \leq C .
$$


(2) $x, y \in \widetilde{E(i)}$ for some $\widetilde{E(i)} \in \mathcal{E}$ and $d(x, y)=1$. We want to show that

$$
d\left(\Pi_{\lambda}(x), \Pi_{\lambda}(y)\right) \leq C .
$$

(3) $x, y \in \widetilde{M_{0}}=X_{\alpha}$ and $d(x, y)=1$. We want to show that

$$
d\left(\Pi_{0}(x), \Pi_{0}(y)\right) \leq C .
$$

(4) $x \in \widetilde{F(i)}=\widetilde{E(i)} \cap \widetilde{M_{0}}$ for some $\widetilde{E(i)} \in \mathcal{E}^{\prime}$. We want to show that

$$
d\left(\Pi_{i}(x), \Pi_{0}(x)\right) \leq C .
$$

(5) $x, y \in \widetilde{F(i)}=\widetilde{E(i)} \cap \widetilde{M_{0}}$ for some $\widetilde{E(i)} \in \mathcal{E}^{\prime}$ and $d(x, y)=1$. We want to show that

$$
d\left(\Pi_{i}(x), \Pi_{i}(y)\right) \leq C .
$$

In the above check-list, the first and fourth steps ensure that there is approximate agreement on $\widetilde{F}_{i}$ for the two possible definitions of $\Pi_{\lambda}$ occurring in the non-definition given earlier. This ensures smooth passage from the non-definition to the definition. The rest of the steps are required to prove the three cases in the definition of $\Pi_{\lambda}$.

Step 1: Bounded discrepancy on $\widetilde{F(i)}$ when $\widetilde{E(i)} \in \mathcal{E} \quad$ The next lemma follows easily from the fact that local quasigeodesics in a hyperbolic metric space are quasigeodesics [19] (See also [36, Lemma 3.1]). If $x, y$ are points in a hyperbolic metric space, $[x, y]$ will denote a geodesic joining them.

Lemma 4.5 Given $\delta, C_{1}>0$, there exist $D, C_{0}$ such that if $a, b, c, d$ are vertices of a $\delta$-hyperbolic metric space $(Z, d)$, and $W \subset Z$ is a $C_{1}$-quasiconvex set, with $d(a, W)=d(a, b), d(d, W)=d(c, d)$ and $d(b, c) \geq D$ then $[a, b] \cup W \cup[c, d]$ is $C_{0}$-quasiconvex. Also, if $[b, c]_{W}$ denotes the shortest path in $W$ joining $b, c$, then $[a, b] \cup[b, c]_{W} \cup[c, d]$ is a $C_{0}$-quasigeodesic. Further, if $[b, c]_{\mathrm{amb}}$ denotes an ambient quasigeodesic in $(Z-W)$ (adding on $b, c$ as the initial and terminal points), then $[a, b] \cup[b, c]_{\mathrm{amb}} \cup[c, d]$ is an ambient $C_{0}$-quasigeodesic in $(Z-W)$.

The next couple of lemmas are taken from [36], where they are stated in the general framework of trees of hyperbolic metric spaces satisfying the qi-embedded condition. $X_{v}, X_{e}, f_{v}$ denote respectively vertex space, edge space and qi-embedding of edge space in vertex space.

Lemma 4.6 [36, Lemma 3.6] Let $X$ be a tree $T$ of hyperbolic metric spaces and $v$ be a vertex of $T$. Let $C>0$. Let $\mu_{1}=[a, b] \subset X_{v}$ be a geodesic and let $e$ be an edge of $T$ incident on $v$. Let $p, q \in N_{C}\left(\mu_{1}\right) \cap f_{v}\left(X_{e}\right)$ be such that $d_{v}(p, q)$ is maximal. Let $\mu_{2}$ be a geodesic in $X_{v}$ joining $p, q$. If $r \in N_{C}\left(\mu_{1}\right) \cap f_{v}\left(X_{e}\right)$, then $d_{v}\left(r, \mu_{2}\right) \leq D_{1}$ for some constant $D_{1}$ depending only on $C, \delta$. 
Proof Let $\pi$ denote a nearest point projection onto $\mu_{1}$. Since $\mu_{2}$ and $[\pi(p), \pi(q)] \subset$ $\mu_{1}$ are geodesics whose end-points lie at distance at most $C$ apart, there exists $C^{\prime}$ such that $[\pi(p), \pi(q)] \subset N_{C^{\prime}}\left(\mu_{2}\right)$. If $\pi(r) \in[\pi(p), \pi(q)]$, then

$$
d\left(r, \mu_{2}\right) \leq C+C^{\prime} .
$$

If $\pi(r) \notin[\pi(p), \pi(q)]$, then without loss of generality, assume $\pi(r) \in[a, \pi(p)] \subset$ $[a, \pi(q)]$. Then

$$
\begin{aligned}
d(p, q) & \geq d(r, q) \\
& \geq d(\pi(r), \pi(q))-2 C \\
& =d(\pi(r), \pi(p))+d(\pi(p), \pi(q))-2 C \\
& \geq d(\pi(r), \pi(p))+d(p, q)-4 C \\
\Rightarrow d(\pi(r), \pi(p)) & \leq 4 C \\
\Rightarrow d(r, p) & \leq 6 C \\
\Rightarrow d\left(r, \mu_{2}\right) & \leq 6 C .
\end{aligned}
$$

Choosing $D_{1}=\max \left\{C+C^{\prime}, 6 C\right\}$, we are through.

Lemma 4.7 [36, Lemma 3.7] Let $\mu_{1}, \mu_{2}$ be as in Lemma 4.6 above. Let $\pi_{i}$ denote nearest point projections onto $\mu_{i}(i=1,2)$. If $p \in f_{v}\left(X_{e}\right)$, then $d\left(\pi_{1}(p), \pi_{2}(p)\right) \leq C_{6}$ for some constant $C_{6}$ depending on $\delta$ alone.

Proof If $d\left(\pi_{1}(p), \pi_{1} \cdot \pi_{2}(p)\right) \leq D$, then $d\left(\pi_{1}(p), \pi_{2}(p)\right) \leq C+D$.

Else, suppose $d\left(\pi_{1}(p), \pi_{1} \cdot \pi_{2}(p)\right)>D$. Then $\left[p, \pi_{1}(p)\right] \cup\left[\pi_{1}(p), \pi_{1} \cdot \pi_{2}(p)\right] \cup$ $\left[\pi_{1} \cdot \pi_{2}(p), \pi_{2}(p)\right]$ is a (uniform) quasigeodesic by Lemma 4.5. But $p, \pi_{2}(p) \in f_{v}\left(X_{e}\right)$ which is quasiconvex. Hence there exists (uniform) $C_{1}$ and $r \in f_{v}\left(X_{e}\right)$ such that $d\left(r, \pi_{1}(p)\right) \leq C_{1}$.

Then, by Lemma 4.6 above, there exists $s \in \mu_{2}$ such that $d\left(s, \pi_{1}(p)\right) \leq C_{1}+D_{1}$. Again (see for instance [36, proof of Lemma 3.2]), $(p, s)_{\pi_{2}(p)} \leq 2 \delta$. Hence,

$$
\left(p, \pi_{1}(p)\right)_{\pi_{2}(p)} \leq 2 \delta+C_{1}+D_{1} .
$$

Similarly, $\left(p, \pi_{1} \cdot \pi_{2}(p)\right)_{\pi_{1}(p)} \leq 2 \delta$. Hence,

$$
\left(p, \pi_{2}(p)\right)_{\pi_{1}(p)} \leq 2 \delta+C .
$$

Therefore, using the definition of Gromov inner product,

$$
d\left(\pi_{1}(p), \pi_{2}(p)\right)=\left(p, \pi_{1}(p)\right)_{\pi_{2}(p)}+\left(p, \pi_{2}(p)\right)_{\pi_{1}(p)} \leq 4 \delta+2 C+D_{1} .
$$


Choosing $C_{6}=4 \delta+2 C+D_{1}$ we are through.

Though we have stated and proved Lemma 4.7 and Lemma 4.6 for geodesic segments, the proof goes through for quasiconvex sets. What Lemma 4.7 effectively says is the following.

We start with a geodesic $\mu_{1}$ in a hyperbolic metric space $X=X_{v} . W=f_{v}\left(X_{e}\right) \subset X_{v}$ is a $C_{1}$-quasiconvex set. We consider the set of points

$$
P=\left\{p \in W: d\left(p . \mu_{1}\right) \leq C_{2}\right\} .
$$

Choose $x, y \in P$ such that $d(x, y)$ is maximal. Let $\mu_{2}$ be the geodesic joining $x, y$. Then nearest-point retractions onto $\mu_{1}, \mu_{2}$ almost agree for points in $W$.

This becomes easier to grasp if all sets in sight are convex subsets of $\mathbb{H}^{n}$. We start with $X=\mathbb{H}^{n} . W \subset X$ is convex. $\mu_{1}$ is replaced by another convex set $V \subset X$. We consider the intersection of neighborhoods $N_{\epsilon}(V) \cap N_{\epsilon}(W)=V_{1} \neq \varnothing$. Then nearest point retractions onto $V_{1}$ and $V$ almost agree for $p \in W$. This is the context of Lemma 4.7 in general. We quasify this below.

Corollary 4.8 Given $\delta, C, C_{1}>0$, there exists $D>0$ such that if

(1) $A \subset Y, B \subset Z, Y \subset Z$ are inclusions of $C_{1}$-quasiconvex sets into $\delta$ hyperbolic metric spaces,

(2) $A$ and $\left.\left(N_{C}(B)\right) \cap Y\right)$ are within a Hausdorff distance of $D$ of each other

(3) $\Pi_{A}$ denote a nearest point projection of $Y$ onto $A$, and $\Pi_{B}$ denote a nearest point projection of $Z$ onto $B$,

then for all $y \in Y, d\left(\Pi_{A}(y), \Pi_{B}(y)\right) \leq D$.

There exist (possibly) curves $\sigma_{i j}$ on $F(i)$ homotopic to some curves on components $\Delta_{j}$ of $P$ in $(M, P)$. Then $\sigma_{i j}$ is one boundary component of a (possibly immersed) annulus $A_{i j}$ whose other boundary component lies on $\Delta_{j}$. Let $J(i)=F(i) \cup \bigcup A_{i j} \subset M_{0}$. Lift $J(i)$ to the universal cover $\widetilde{M_{0}}$ to get copies of $\widetilde{J(i)}$.

We will show that on $\widetilde{F(i)}$, the two putative definitions of $\Pi_{\lambda}$ almost agree. Suppose $\mu_{i}=\left[a_{i}, b_{i}\right]_{F} \subset \widetilde{F(i)} \subset \widetilde{G(i)}$ is a geodesic in the path-metric on $\widetilde{F(i)}$. As in the construction of $B_{0}(\lambda)$, we can construct a 'hyperbolic' geodesic $\mu_{i}^{h}$ joining $a_{i}, b_{i}$ in $\widetilde{G(i)}$. Such a geodesic has unique entry and exit points for every horodisk and hence unique 'Euclidean' geodesics joining them on the bounding horocycles. Replacing the hyperbolic segments by Euclidean segments, we obtain an ambient quasigeodesic $\mu_{i}^{a}$ 
(by Theorem 2.7) in $\widetilde{J(i)}$. For $x \in \widetilde{F(i)}$, let $\Pi_{i 1}$ denote a nearest point retraction onto $\mu_{i}$ in the path metric on $\widetilde{F(i)}$. Also, let $\Pi_{i 2}$ denote a nearest point retraction onto $\mu_{i}^{a} \subset \widetilde{J(i)}$ in the path metric on $\widetilde{J(i)}$. Then from Lemma 4.6 and Lemma 4.7 above, we get the following lemma.

Lemma 4.9 There exists $C>0$ such that for all $i$ and $x \in \widetilde{F(i)} \subset \widetilde{J(i)}$,

$$
d\left(\Pi_{i 1}(x), \Pi_{i 2}(x)\right) \leq C .
$$

Again, as in the construction of $B_{0}(\lambda)$, each of the Euclidean geodesic segments in $\mu_{i}^{a}$ along with the hyperbolic geodesic segments of $\mu_{i}^{h}$ joining its end-points (and lying entirely within the corresponding horodisk) bounds a totally geodesic disk. The union of $\mu_{i}^{a}$ and all these totally geodesic disks is denoted by $B_{0}\left(\mu_{i}\right)$. There exists $C_{1}>0$ such that each $B_{0}\left(\mu_{i}\right)$ is $C_{1}$-quasiconvex in $\widetilde{G_{i}}$ and hence in $\widetilde{M_{0}}$. (See for instance McMullen [26, Section 8].)

Let $\Pi_{i 3}$ denote a nearest point projection onto $B_{0}\left(\mu_{i}\right)$, in $\widetilde{G(i)}$.

Lemma 4.10 There exists $C>0$ such that for all $i$ and $x \in \widetilde{F(i)} \subset \widetilde{J(i)}$,

$$
d\left(\Pi_{i 2}(x), \Pi_{i 3}(x)\right) \leq C .
$$

Proof For the purposes of this lemma, $[a, b]$ will denote a geodesic in $\widetilde{G(i)}$. First, $\left[x, \Pi_{i 2}(x)\right] \cup \mu_{i}^{a}$ is a tripod in $\widetilde{J(i)}$ and hence it is uniformly quasiconvex from Lemma 4.5. Again, by the same lemma, $\left[x, \Pi_{i 3}(x)\right] \cup B_{0}\left(\mu_{i}\right)$ is quasiconvex. Further, $\Pi_{i 3}(x) \in \mu_{i}^{a}$ (since $\mu_{i}^{a}$ forms the boundary of $B_{0}\left(\mu_{i}\right)$ in $\widetilde{G(i)}$ and separates it from the $\widetilde{J(i)})$. Therefore, $\left[x, \Pi_{i 2}(x)\right] \cup\left[\Pi_{i 2}(x), a_{i}\right]$ and (from Theorem 2.7) $\left[x, \Pi_{i 3}(x)\right] \cup$ $\left[\Pi_{i 3}(x), a_{i}\right]$ are both ambient quasigeodesics in the hyperbolic metric space $\widetilde{G(i)}$. In the same way, $\left[x, \Pi_{i 2}(x)\right] \cup\left[\Pi_{i 2}(x), b_{i}\right]$ and $\left[x, \Pi_{i 3}(x)\right] \cup\left[\Pi_{i 3}(x), b_{i}\right]$ are both ambient quasigeodesics in the hyperbolic metric space $\widetilde{G(i)}$.

Hence, $\Pi_{i 3}(x)$ must lie in a bounded neighborhood of $\left[x, \Pi_{i 2}(x)\right] \cup\left[\Pi_{i 2}(x), a_{i}\right]$, as also $\left[x, \Pi_{i 2}(x)\right] \cup\left[\Pi_{i 2}(x), b_{i}\right]$, as also $\mu_{i}^{a}$. Hence, it must lie close to the intersection of these three sets, which is $\Pi_{i 2}(x)$. This proves the lemma.

Recall that $\Pi_{0}$ denotes nearest point projection onto $B_{0}(\lambda)$. Now, $B_{0}\left(\mu_{i}\right)$ lies in a bounded neighborhood of $B_{0}(\lambda)$, since $\mu_{i}$ lies in a bounded neighborhood of $\lambda$. Now from Corollary 4.8, choosing $Y=\widetilde{G(i)}, Z=\widetilde{M_{0}}, A=B_{0}\left(\mu_{i}\right), B=B_{0}(\lambda)$, we obtain the following corollary. 
Corollary 4.11 There exists $C>0$ such that for all $x \in \widetilde{F(i)} \subset \widetilde{J(i)} \subset \widetilde{G(i)} \subset \widetilde{M_{0}}$, $d\left(\Pi_{0}(x), \Pi_{i 3}(x)\right) \leq C$.

Combining Lemma 4.9, Lemma 4.10 and Corollary 4.11, we get the following lemma.

Lemma 4.12 Recall that for $x \in \widetilde{F(i)}, \Pi_{i}$ denotes nearest point retraction onto $\mu_{i}$ and $\Pi_{0}$ denotes nearest point retraction onto $B_{0}(\lambda)$. Then $d\left(\Pi_{i}(x), \Pi_{\alpha}(x) \leq C_{4}\right.$ for some $C_{4} \geq 0$.

Step 2: Retract on $\widetilde{E(i)}$ for $\widetilde{E(i)} \in \mathcal{E} \quad$ A number of lemmas will be necessary. These are lifted directly from [36]. We state them here without proof.

The following lemma says nearest point projections in a $\delta$-hyperbolic metric space do not increase distances much.

Lemma 4.13 [36, Lemma 3.2] Let $(Y, d)$ be a $\delta$-hyperbolic metric space and let $\mu \subset Y$ be a $C$-quasiconvex subset, eg a geodesic segment. Let $\pi: Y \rightarrow \mu$ map $y \in Y$ to a point on $\mu$ nearest to $y$. Then $d(\pi(x), \pi(y)) \leq C_{3} d(x, y)$ for all $x, y \in Y$ where $C_{3}$ depends only on $\delta, C$.

The following lemma says that nearest point projections and quasi- isometries in hyperbolic metric spaces 'almost commute'.

Lemma 4.14 [36, Lemma 3.5] Suppose $(Y, d)$ is $\delta$-hyperbolic. Let $\mu_{1}$ be some geodesic segment in $Y$ joining $a, b$ and let $p$ be any vertex of $Y$. Also let $q$ be a vertex on $\mu_{1}$ such that $d(p, q) \leq d(p, x)$ for any $x \in \mu_{1}$. Let $\phi$ be a $(K, \epsilon)$-quasi-isometry from $Y$ to itself. Let $\mu_{2}$ be a geodesic segment in $Y$ joining $\phi(a)$ to $\phi(b)$. Let $r$ be a point on $\mu_{2}$ such that $d(\phi(p), r) \leq d(\phi(p), x)$ for $x \in \mu_{2}$. Then $d(r, \phi(q)) \leq C_{4}$ for some constant $C_{4}$ depending only on $K, \epsilon$ and $\delta$.

Theorem 4.15 There exists $C>0$, such that if $x, y \in \widetilde{E(i)}$ for some $\widetilde{E(i)} \in \mathcal{E}$, and $d(x, y)=1$, then $d\left(\Pi_{i}(x), \Pi_{i}(y)\right) \leq C$.

Proof Case (a) $\quad x, y \in X_{i j}$ for some $i=1, \ldots, s, j=0,1, \ldots$ This follows directly from Lemma 4.13.

Case (b) $\quad x \in X_{i, j-1}$ and $y \in X_{i j}$ for some $i=1, \ldots, s, j=0,1, \ldots$ Recall that $\mu_{i k}=B\left(\mu_{i}\right) \cap X_{i k}$ for all $i, k$. Then $\Pi_{i}(z)=\Pi_{i k}(x)$ for $z \in X_{i k}$. Also, from Lemma 3.9, there exist $K, \epsilon>0$ such that for all $i, k, \phi_{i k}$ is a $(K, \epsilon)$-quasi-isometry. Hence, $\phi_{i j}\left(\mu_{i, j-1}\right)$ is a $(K, \epsilon)$-quasigeodesic lying in a bounded $K^{\prime}$-neighborhood of $\Phi_{i j}\left(\mu_{i, j-1}\right)=\mu_{i j}$ where $K^{\prime}$ depends only on $K, \epsilon, \delta$. 
Now $\phi_{i j} \circ \Pi_{i, j-1}(x)$ lies on this quasi-geodesic. By Lemma 4.14, there exists $C_{0}>0$ such that $d\left(\phi_{i j} \circ \Pi_{i, j-1}(x), \Pi_{i j} \circ \phi_{i, j-1}(x)\right) \leq C_{0}$. Also $d(x, y)=1=d\left(x, \phi_{i j}(x)\right)$. Hence, $d\left(\phi_{i j}(x), y\right) \leq 2$ and $\phi_{i j}(x), y \in X_{i j}$. Therefore, by Lemma 4.13, there exists $C_{1}$ such that $d\left(\Pi_{i j} \circ \phi_{i j}(x), \Pi_{i j}(y)\right) \leq 2 C_{1}$. Hence

$$
\begin{aligned}
d\left(\Pi_{i}(x), \Pi_{i}(y)\right)= & d\left(\Pi_{i, j-1}(x), \Pi_{i j}(y)\right) \\
\leq & d\left(\Pi_{i, j-1}(x), \phi_{i j} \circ \Pi_{i, j-1}(x)\right)+ \\
& d\left(\phi_{i j} \circ \Pi_{i, j-1}(x), \Pi_{i j} \circ \phi_{i, j-1}(x)\right)+d\left(\Pi_{i j} \circ \phi_{i j}(x), \Pi_{i j}(y)\right) \\
\leq & 1+C_{0}+2 C_{1} .
\end{aligned}
$$

Choosing $C=\left(1+C_{0}+2 C_{1}\right)$, we are through.

Step 3 Retract on $\widetilde{M_{0}}=X_{\alpha}$. This case follows directly from Lemma 4.13. We state a special case required for our specific purposes.

Lemma 4.16 There exists $C>0$ such that if $x, y \in \widetilde{M_{0}}=X_{\alpha}$ and $d(x, y)=1$, then $d\left(\Pi_{0}(x), \Pi_{0}(y)\right) \leq C$.

Step 4 Bounded discrepancy on $\widetilde{F(i)}$ when $\widetilde{E(i)} \in \mathcal{E}^{\prime}$. We want to show that for $x, y \in \widetilde{F(i)} \subset \widetilde{E(i)} \in \mathcal{E}^{\prime}, d\left(\Pi_{0}(x), \Pi_{i}(x)\right)$ is uniformly bounded.

Recall from the definition of $\widetilde{G(i)}$, two cases may arise.

Case (a) $\quad N_{2 k}(\widetilde{G(i)})$ does not intersect $\mathcal{H}(\lambda)$. In this case, recall that $y \in \widetilde{F(i)}$ is chosen arbitrarily, and we had defined

$$
\Pi_{i}(x)=\Pi_{0}(y) \text { for all } x \in \widetilde{E} .
$$

Next, by the choice of $K=3 k+4 \delta+k^{\prime}$ in the definition of $\mathcal{E}^{\prime}$, and Corollary 4.4, we have that the diameter of the set $\Pi_{0}(\widetilde{G(i)})$ and hence $\Pi_{0}(\widetilde{F(i)})$ is bounded by $k$ (from Lemma 4.2).

Hence, $d\left(\Pi_{0}(x), \Pi_{i}(x)\right)=d\left(\Pi_{0}(x), \Pi_{0}(y)\right) \leq k$.

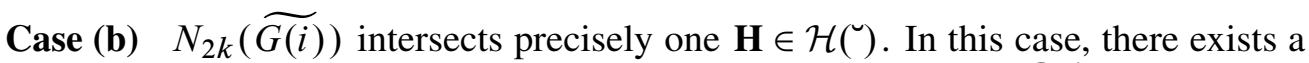
unique lift $\widetilde{\sigma}$ of a curve $\sigma$ on $F(i)$, (parabolic in $M_{0}$ ) lying on $\widetilde{F(i)}$ at a bounded distance from $\mathbf{H}$. Recall that $\Pi_{\sigma}$ denotes nearest point projection of $\widetilde{E(i)}$ onto $\widetilde{\sigma}$ and $\Pi_{\sigma}^{0}(x)$ denotes nearest point projection onto $B(\widetilde{\sigma})$. On $\widetilde{F(i)}, \Pi_{\sigma}^{0}$ coincides with $\Pi_{\sigma} \circ \Pi_{\sigma}^{0}$. Also recall that $\Pi_{i}(x)=\Pi_{0} \circ \Pi_{\sigma} \circ \Pi_{\sigma}^{0}(x)$ for all $x \in \widetilde{E(i)}$.

On $\widetilde{F(i)}, \Pi_{\sigma}^{0}$ coincides with $\Pi_{\sigma} \circ \Pi_{\sigma}^{0}$. Thus, we want to show that $d\left(\Pi_{0}(x), \Pi_{0} \circ\right.$ $\left.\Pi_{\sigma}^{0}(x)\right)$ is uniformly bounded on $\widetilde{F(i)}$. 
Here, $N_{k}(\widetilde{G(i)}) \cap N_{k}\left(B_{0}(\lambda)\right)$ lies in some horoball $\mathbf{H} \in \mathcal{H}(\widetilde{)})$ while $N_{k}(\widetilde{F(i)}) \cap$ $N_{k}\left(B_{0}(\lambda)\right)=\varnothing\left(\right.$ as per definition of $\left.\mathcal{E}^{\prime}\right)$. By Lemma $4.2 \Pi_{0}(\widetilde{F(i)}) \subset N_{k}(\widetilde{(A \cup \kappa)} \subset$ $N_{k}(\mathbf{H})$, where $\kappa \subset G(i)$ is the cusp whose boundary $\eta$ bounds the annulus $A$ along with $\sigma$ in $M_{0}$. (Recall from the discussion following Lemma 4.2 that each $\eta$ forms the boundary of a totally geodesic 2 dimensional subset $\kappa$ of a $Z$ cusp or a $Z+Z$-cusp of $M_{0}$.) Also, $\widetilde{(A \cup \kappa)}=\widetilde{A_{\kappa}}$ (say) is $C_{3}$-quasiconvex for some $C_{3}$.

Let $\Pi_{\kappa}$ denote nearest point projection of $\widetilde{G(i)}$ onto $\widetilde{A_{\kappa}}$ in the metric on $\widetilde{M_{0}}$. Also, let $\Pi_{0}^{\text {aug }}$ denote nearest point projection onto $B_{0}^{\text {aug }}(\lambda)$. Then by Corollary 4.8 there exists $C_{4}>0$ such that

$$
d\left(\Pi_{\kappa}(x), \Pi_{0}^{\mathrm{aug}}(x)\right) \leq C_{4} \text { for all } x \in \widetilde{G(i)} .
$$

Next, $B_{0}(\lambda) \subset B_{0}^{\text {aug }}(\lambda) \subset \widetilde{M_{0}}$ and both $B_{0}(\lambda)$ and $B_{0}^{\text {aug }}(\lambda)$ are $C_{1}$-quasiconvex. Both $\Pi_{0}$ and $\Pi_{0} \circ \Pi_{0}^{\text {aug }}$ are large-scale Lipschitz retracts onto $B_{0}(\lambda)$, the only difference being that the latter factors through a large-scale Lipschitz retract $\Pi_{0}^{\text {aug }}$ onto $B_{0}^{\text {aug }}(\lambda)$. Hence the two maps must send $x$ to close by points, ie there exists $C_{5}>0$ such that

$$
d\left(\Pi_{0} \circ \Pi_{0}^{\mathrm{aug}}(x), \Pi_{0}(x)\right) \leq C_{5} \text { for all } x \in \widetilde{M_{0}} .
$$

Using the above two inequalities along with Lemma 4.13 we get a $C_{6}>0$ such that

$$
d\left(\Pi_{0} \circ \Pi_{\kappa}(x), \Pi_{0}(x)\right) \leq C_{6} \text { for all } x \in \widetilde{M_{0}} .
$$

Finally, we observe that for $x \in \widetilde{F(i)}$, since $\Pi_{\sigma}^{0}$ denotes nearest point projection onto $\widetilde{\sigma}$, then $d\left(\Pi_{\kappa}(x), \Pi_{\sigma}^{0}(x)\right)$ is bounded. This is because $\widetilde{\sigma}$ separates $\widetilde{G(i)}$ into $\widetilde{F(i)}$ and $\widetilde{A_{\kappa}}$. Hence, the geodesic joining $x$ to $\Pi_{\kappa}(x)$ in $\widetilde{G(i)}$ must cut $\widetilde{\sigma}$ at some point which therefore must coincide with $\Pi_{\sigma}^{0}(x)$, provided we take nearest point projections in $\widetilde{G(i)}$. Since $\widetilde{G(i)}$ is quasiconvex in $\widetilde{M_{0}}$, therefore by Corollary 4.8 , we might as well take projections in the $\widetilde{M_{0}}$ metric and we have a $C_{7}>0$ such that

$$
d\left(\Pi_{\kappa}(x), \Pi_{\sigma}^{0}(x)\right) \leq C_{7} \text { for all } x \in \widetilde{F(i)} .
$$

Combining the above two inequalities and using Lemma 4.13 for $\Pi_{0}$, we obtain finally the next lemma.

Lemma 4.17 There exists $C>0$ such that

$$
d\left(\Pi_{0} \circ \Pi_{\sigma}^{0}(x), \Pi_{0}(x)\right) \leq C \text { for all } x \in \widetilde{F(i)} .
$$

Since on such a $\widetilde{F(i)}, \Pi_{0} \circ \Pi_{\sigma}^{0}$ is denoted by $\Pi_{i}$, we have $d\left(\Pi_{i}(x), \Pi_{0}(x)\right) \leq C$ for all $x \in \widetilde{F(i)}$. 
The three results Lemma 4.12, Case (a) of Step 4 above and Lemma 4.17 show that $\Pi_{0}$ and $\Pi_{i}$ almost agree, ie have at most a bounded amount of discrepancy for all $\widetilde{F(i)}$. We summarize these three in the following useful proposition.

Proposition 4.18 There exists $C>0$ such that for all $\widetilde{F(i)}$ and all $x \in \widetilde{F(i)}$,

$$
d\left(\Pi_{i}(x), \Pi_{0}(x)\right) \leq C .
$$

Step 5 Retract on $\widetilde{E(i)}$ for $\widetilde{E(i)} \in \mathcal{E}^{\prime} . \Pi_{i}(x)=\Pi_{0} \circ \Pi_{\sigma} \circ \Pi_{\sigma}^{0}(x)$ for all $x \in \widetilde{E(i)}$. By the proof of Theorem 4.15, there exists $C_{1}>0$ such that for all $x, y \in \widetilde{E(i)} \in \mathcal{E}^{\prime}$ with $d(x, y)=1$, we have

$$
d\left(\Pi_{\sigma}^{0}(x), \Pi_{\sigma}^{0}(y)\right) \leq C_{1} .
$$

(Though Theorem 4.15 is stated for $\widetilde{E(i)} \in \mathcal{E}$, all that we need for the above assertion is the intrinsic metric on $\widetilde{E(i)}$ and here the proof is the same.)

Also, by Lemma 4.13 , there exists $C_{2}>0$ such that for $x, y \in \widetilde{M_{0}}$,

$$
d\left(\Pi_{0}(x), \Pi_{0}(y)\right) \leq C_{2} d(x, y) .
$$

Hence, it would be enough to prove that there exists $C_{3}>0$ such that

$$
d\left(\Pi_{\sigma}(x), \Pi_{\sigma}(y)\right) \leq C_{3} d(x, y)
$$

for all $x, y \in \widetilde{E(i)}$.

For this, it would suffice to show that $\widetilde{\sigma}$ is quasiconvex in $\widetilde{E(i)}$. Recall that there exists a simply degenerate end $E^{h}$, one of whose lifts to the universal cover is $\widetilde{E^{h}(i)} . \widetilde{E^{h}(i)}$ with cusps removed is $\widetilde{E(i)}$. We shall show that $\widetilde{\sigma}$ is quasiconvex in $\widetilde{E^{h}(i)}$. We give $E^{h}$ a convex hyperbolic structure by taking the cover of $N^{h}$ corresponding to $\pi_{1}\left(E^{h}\right)$ and looking at its convex core. This makes the universal cover of $E^{h}$ (equipped with such a convex hyperbolic structure) quasi-isometric to $\widetilde{E^{h}(i)}$. For the purposes of this step, therefore, we assume that $\widetilde{E^{h}(i)}$ is the universal cover of a simply degenerate hyperbolic manifold (so as to avoid the extra expository complication of mapping via quasi-isometries). Now, $\sigma \subset E^{h}$ is a closed curve, and is therefore homotopic by a bounded homotopy to a closed geodesic $\sigma^{\prime}$. Then any lift $\widetilde{\sigma^{\prime}}$ is a hyperbolic geodesic in the convex hyperbolic manifold $\widetilde{E^{h}(i)}$. Since $\widetilde{\sigma}$ lies at a bounded distance from $\widetilde{\sigma^{\prime}}$, we conclude that $\widetilde{\sigma}$ is quasiconvex in $\widetilde{E^{h}(i)}$ and hence there exists a nearest point projection of $\widetilde{E^{h}(i)}$ onto $\widetilde{\sigma}$ which stretches distances by a bounded factor by Lemma 
4.13. Restricting this map to $\widetilde{E(i)}$, the retraction property persists. Since the path metric on $\widetilde{E(i)}$ dominates the metric induced from $\widetilde{E^{h}(i)}$, and since the metric on $\widetilde{\sigma}$ remains undisturbed on removing cusps, we find (reverting to our description of spaces as graphs) that there exists $C_{3}>0$ such that

$$
d\left(\Pi_{\sigma}(x), \Pi_{\sigma}(y)\right) \leq C_{3} d(x, y)
$$

for all $x, y \in \widetilde{E(i)}$.

Combining the three above equations, we arrive at the following proposition.

Proposition 4.19 There exists $C>0$, such that if $x, y \in \widetilde{E(i)}$ for some $\widetilde{E(i)} \in \mathcal{E}^{\prime}$, and $d(x, y)=1$, then $d\left(\Pi_{i}(x), \Pi_{i}(y)\right) \leq C$.

We next prove that $\Pi_{\lambda}$ is a retract. We combine the five steps above to conclude that $\Pi_{\lambda}$ is a retract. Recall the definition of $\Pi_{\lambda}$.

\section{Definition}

$$
\begin{aligned}
\Pi_{\lambda}(x) & =\Pi_{i}(x), x \in \widetilde{E(i)}-X_{i 0}, \widetilde{E(i)} \in \mathcal{E} \\
& =\Pi_{0}(x), x \in X_{\alpha} \\
& =\Pi_{i}(x), x \in \widetilde{E(i)}-X_{i 0}, \widetilde{E(i)} \in \mathcal{E}^{\prime}
\end{aligned}
$$

Also recall that $\widetilde{N_{0}}$ is quasi-isometric to a graph $X$ which can be regarded as a tree of hyperbolic metric spaces with possibly exceptional vertex $\alpha$ satisfying the qi-embedded condition. (See Lemma 3.9, Lemma 3.10 and the discussion preceding Lemma 3.9 where this is elaborated.)

We are now in a position to prove our main technical theorem.

Theorem 4.20 There exists $C_{0} \geq 0$ such that $d\left(\Pi_{\lambda}(x), \Pi_{\lambda}(y)\right) \leq C_{0} d(x, y)$ for $x, y$ vertices of $X$.

Proof It suffices to prove the theorem when $d(x, y)=1$.

(1) For $x, y \in \widetilde{E(i)}-\widetilde{M_{0}}, \widetilde{E(i)} \in \mathcal{E}$, this follows from Theorem 4.15 in Step 2 above.

(2) For $x, y \in X_{\alpha}=\widetilde{M_{0}}$, this follows from Lemma 4.16 in Step 3 above.

(3) For $x, y \in \widetilde{E(i)}-\widetilde{M_{0}}, \widetilde{E(i)} \in \mathcal{E}^{\prime}$, this follows from Proposition 4.19 in Step 5 above.

(4) $x \in X_{\alpha}, y \in X_{i 1} \subset \widetilde{E(i)}$ and $d(x, y)=1$ for $\widetilde{E(i)} \in \mathcal{E} \cup \mathcal{E}^{\prime}$. 
Here,

$$
\begin{aligned}
d\left(\Pi_{\lambda}(x), \Pi_{\lambda}(y)\right) & =d\left(\Pi_{0}(x), \Pi_{i}(y)\right) \\
& \leq d\left(\Pi_{0}(x), \Pi_{i}(x)\right)+d\left(\Pi_{i}(x), \Pi_{i}(y)\right) .
\end{aligned}
$$

Choose constants $C_{1}, C_{2}$ and $C_{3}$ from Proposition 4.18, Theorem 4.15 and Proposition 4.19. Let $C=C_{1}+\max \left(C_{2}, C_{3}\right)$. Then we get

$$
d\left(\Pi_{\lambda}(x), \Pi_{\lambda}(y)\right) \leq C .
$$

This proves the result.

\section{Proof of main theorem}

Recall that we started off with a geodesic $\lambda^{h}$ on $\widetilde{M_{\mathrm{gf}}}$ outside an $n$-ball around a fixed reference point $p$, joining $a, b$. Replacing the maximal segments of $\lambda^{h}$ lying inside $Z$-horoballs by 'Euclidean' geodesics lying on the corresponding horosphere, we obtained an ambient quasigeodesic $\lambda$ in $\widetilde{M_{0}}$ joining $a, b . \lambda$ agrees with $\lambda^{h}$ off $Z$-horoballs. Let $\beta^{h}$ be the geodesic in $\widetilde{N^{h}}$ joining $a, b$. Performing the same operation on $\beta^{h}$ for $Z$-horoballs in $\widetilde{N^{h}}$, we obtain an ambient geodesic $\beta_{\text {amb }}^{0}$ in $\widetilde{N_{0}}$ joining the end-points of $\lambda$. We have proved the existence of a retraction $\Pi_{\lambda}$ in the preceding section (Theorem 4.20). Project $\beta_{\mathrm{amb}}^{0}$ onto $B_{\lambda}$, using $\Pi_{\lambda}$ to get another ambient quasigeodesic $\beta_{\mathrm{amb}}$. Thus, $\beta_{\mathrm{amb}}=\Pi_{\lambda}\left(\beta_{\mathrm{amb}}^{0}\right)$.

Our starting point for this section is therefore the ambient quasigeodesic $\beta_{\text {amb }} \subset B_{\lambda} \subset$ $\widetilde{N_{0}}$.

\subsection{Quasigeodesic rays}

The purpose of this subsection is to construct for any $\widetilde{E(i)} \in \mathcal{E}$ and $x \in B\left(\mu_{i}\right)=$ $B_{\lambda} \cap \widetilde{E(i)}$ a quasi-geodesic ray $r_{x} \subset B_{\lambda}$ passing through $x . r_{x}$ can be regarded both as a function $r_{x}:\{0\} \cup \mathbb{N} \rightarrow B\left(\mu_{i}\right)$ or as a subset of $B\left(\mu_{i}\right)$ (the image of the function $r_{x}$ ). If $x$ lies away from cusps, so will $r_{x}$.

Fix $\widetilde{E} \in \mathcal{E}$. Recall that

- $\widetilde{E} \cap \widetilde{M_{0}}=\widetilde{F}$,

- $\widetilde{F} \cap B_{\lambda}=\mu=\mu_{0}$,

- $\widetilde{E}=\bigcup_{i} \widetilde{S(i)}$,

- $B(\mu)=\cup_{i} \mu_{i}$, 
- $\mu_{i}=\Phi_{i}\left(\mu_{i-1}\right) \subset \widetilde{S(i)}$

Also note that $\Phi_{i}$ is the map on geodesics induced by the quasi-isometry $\phi_{i}: \overline{S(i-1)} \rightarrow$ $\widetilde{S(i)}$. The quasi-isometry $\phi_{i}$ is induced by a cusp-preserving homeomorphism of truncated pleated surfaces. So we can assume that $\phi_{i}$ restricted to each horocycle boundary component of $\overline{S(i-1)}$ is an orientation-preserving isometry. Since each such horocycle may naturally be identified with $\mathbb{R}$, we might as well assume that the map on horocycles induced by $\phi_{i}$ is the identity map. Now, extend $\phi_{i}$ to a map $\phi_{i}^{h}: \widetilde{S^{h}(i-1)} \rightarrow \widetilde{S^{h}(i)}$ by demanding that $\phi_{i}^{h}$ restricted to each horodisk is an isometry. Note that $\phi_{i}^{h}$ is thus an equivariant quasi-isometry from $\mathbb{H}^{2}$ to itself, taking horodisks isometrically to horodisks. Next, let $\mu_{i}^{h}$ be the hyperbolic geodesic joining the end-points of $\mu_{i}$. As usual, let $\mu_{i}^{a}$ denote the ambient quasigeodesic obtained by replacing hyperbolic segments in horodisks by 'Euclidean' segments on the boundary horocycles. Then $\mu_{i}^{a}$ lies in a uniform (independent of $i$ ) $C_{0}$-neighborhood of $\mu_{i}$ as they are both ambient quasigeodesics in $\widetilde{S(i)}$. Let

$$
B^{a}(\mu)=\cup_{i} \mu_{i}^{a}
$$

Then $B^{a}(\mu)$ lies in a $C_{0}$-neighborhood of $B(\mu)$. Let $\mu_{i}^{c}$ denote the union of the segments of $\mu_{i}^{a}$ which lie along horocycles and let $\mu_{i}^{b}=\mu_{i}^{a}-\mu_{i}^{c}$. Let

$$
\begin{aligned}
& B^{c}(\mu)=\cup_{i} \mu_{i}^{c} \\
& B^{b}(\mu)=\cup_{i} \mu_{i}^{b} .
\end{aligned}
$$

We want to show that for all $x \in B^{b}(\mu)$ there exists a $C$-quasigeodesic $r_{x}:\{0\} \cup \mathbb{N} \rightarrow$ $B^{b}(\mu)$ such that $x \in r_{x}(\{0\} \cup \mathbb{N})$ and $r_{x}(i) \in \mu_{i}^{b}$. Suppose $x \in \mu_{k}^{b} \subset B^{b}(\mu)$. We define $r_{x}$ by starting with $r_{x}(k)=x$ and construct $r_{x}(k-i)$ and $r_{x}(k+i)$ inductively (of course $(k-i)$ stops at 0 but $(k+i)$ goes on to infinity). For the sake of concreteness, we prove the existence of such a $r_{x}(k+1)$. The same argument applies to $(k-1)$ and inductively for the rest.

Lemma 5.1 There exists $C>0$ such that if $r_{x}(k)=x \in \mu_{k}^{b}$ then there exists $x^{\prime} \in \mu_{k+1}^{b}$ such that $d\left(x, x^{\prime}\right) \leq C$. We denote $r_{x}(k+1)=x^{\prime}$.

Proof Let $[a, b]$ be the maximal connected component of $\mu_{k}^{b}$ on which $x$ lies. Then there exist two horospheres $\mathbf{H}_{\mathbf{1}}$ and $\mathbf{H}_{\mathbf{2}}$ such that $a \in \mathbf{H}_{\mathbf{1}}$ (or is the initial point of $\mu_{k}$ ) and $b \in \mathbf{H}_{2}$ (or is the terminal point of $\mu_{k}$ ). Note that $[a, b]$ does not intersect any of the horodisks of $\widetilde{S_{k}^{h}}$. Since $\phi_{k+1}^{h}$ preserves horodisks, $\phi_{k+1}(a)$ lies on a horocycle (or is the initial point of $\mu_{k+1}$ ) as does $\phi_{k+1}(b)$ (or is the terminal point 
of $\mu_{k+1}$ ). Further, the image of $[a, b]$ is a hyperbolic quasigeodesic (which we now denote as $\left.\phi_{k+1}([a, b])\right)$ lying outside horoballs. Let $\Phi_{k+1}^{h}([a, b])$ denote the hyperbolic geodesic joining $\phi_{k+1}(a)$ and $\phi_{k+1}(b)$. Let $\Phi_{k+1}([a, b])$ denote the ambient geodesic in $\widehat{S(k+1)}$ joining $\phi_{k+1}(a)$ and $\phi_{k+1}(b)$. Therefore $\Phi_{k+1}^{h}([a, b])$ lies in a bounded neighborhood of $\phi_{k+1}([a, b])$ (which in turn lies at a bounded distance from $\left.\Phi_{k+1}([a, b])\right)$ and hence by Theorem 2.8 there exists an upper bound on how much $\Phi_{k+1}^{h}([a, b])$ can penetrate horoballs, ie there exists $C_{1}>0$ such that for all $z \in \Phi_{k+1}^{h}([a, b])$, there exists $z^{\prime} \in \Phi_{k+1}^{h}([a, b])$ lying outside horoballs with $d\left(z, z^{\prime}\right) \leq C_{1}$. Further, since $\phi_{k+1}$ is a quasi-isometry there exists $C_{2}>0$ such that $d\left(\phi_{k+1}(x), \Phi_{k+1}^{h}([a, b])\right) \leq C_{2}$. Hence there exists $x^{\prime} \in \Phi_{k+1}^{h}([a, b])$ such that

- $d\left(\phi_{k+1}(x), x^{\prime}\right) \leq C_{1}+C_{2}$,

- $x^{\prime}$ lies outside horoballs.

Again, $\Phi_{k+1}([a, b])$ lies at a uniformly bounded distance $\leq C_{3}$ from $\mu_{k+1}$ and so, if $c, d \in \mu_{k+1}$ such that $d(a, c) \leq C_{3}$ and $d(b, d) \leq C_{3}$ then the segment $[c, d]$ can penetrate only a bounded distance into any horoball. Hence there exists $C_{4}>0$ and $x^{\prime \prime} \in[c, d]$ such that

- $d\left(x^{\prime}, x^{\prime \prime}\right) \leq C_{4}$,

- $x^{\prime \prime}$ lies outside horoballs.

Hence, $d\left(\phi_{k+1}(x), x^{\prime \prime}\right) \leq C_{1}+C_{2}+C_{4}$. Since $d\left(x, \phi_{k+1}(x)\right)=1$, we have, by choosing $r_{k+1}(x)=x^{\prime \prime}$,

$$
d\left(r_{k}(x), r_{k+1}(x)\right) \leq 1+C_{1}+C_{2}+C_{4} .
$$

Choosing $C=1+C_{1}+C_{2}+C_{4}$, we are through.

Using Lemma 5.1 repeatedly (inductively replacing $x$ with $r_{x}(k+i)$ we obtain the values of $r_{x}(i)$ for $i \geq k$. By an exactly similar symmetric argument, we get $r_{x}(k-1)$ and proceed down to $r_{x}(0)$. Now for any $i, z \in \widetilde{S(i)}$ and $y \in \widetilde{S(i+1)}, d(z, y) \geq 1$. Hence, for any $z \in \widetilde{S(i)}$ and $y \in \widetilde{S(j)}, d(z, y) \geq|i-j|$. This gives the following corollary.

Corollary 5.2 There exist $K, \epsilon>0$ such that for all $x \in \mu_{k}^{b} \subset B^{b}(\mu)$ there exists a $(K, \epsilon)$ quasigeodesic ray $r_{x}$ such that $r_{x}(k)=x$ and $r_{x}(i) \in \mu_{i}^{b}$ for all $i=0,1,2, \ldots$ 


\section{2 p-incompressible boundary}

Till this point we have not used the hypothesis of $p$-incompressibility. We need a modification of Corollary 5.2 above to go down from a point in $\mu^{b}=\mu_{0}^{b}$ to a point in $\lambda^{b}$.

We can change $B_{\lambda}$ to $B_{\lambda}^{a}$ by replacing each $B(\mu) \subset \widetilde{E(i)}$ by $B^{a}(\mu)$ for $\widetilde{E(i)} \in \mathcal{E}$. Recall that we had started off with $\lambda$ being an ambient quasigeodesic in $\widetilde{M_{0}}$ constructed from $\lambda^{h}$ by replacing hyperbolic geodesic segments within $Z$-horoballs by 'Euclidean' geodesic segments along horospheres. So, there is no need to freshly construct a $\lambda^{a}$ (we can think that $\lambda=\lambda^{a}$ ). But we do need to construct $\lambda_{\text {cc }}$ by performing the same modifications for $(Z+Z)$-horoballs. Just as in the construction of $\mu_{i}^{a}, \mu_{i}^{c}, \mu_{i}^{b}$, we can define $\lambda^{c}$ to be the collection of subsegments of $\lambda_{\text {cc }}$ lying along horoballs ( $Z$ or $(Z+Z))$, and $\lambda^{b}=\lambda_{\mathrm{cc}}-\lambda^{c}$. Adjoining $\lambda_{\mathrm{cc}}, \lambda^{c}$ and $\lambda^{b}$ to $B^{a}(\mu), B^{c}(\mu)$ and $B^{b}(\mu)$ we get respectively, $B^{a}(\mu, \lambda), B^{c}(\mu, \lambda)$ and $B^{b}(\mu, \lambda)$. Then we can extend Corollary 5.2 above so that $\lambda$ is included.

For the convenience of the reader, we summarize the notation for the different types of geodesics and quasigeodesics that we have introduced and will use henceforth.

- $\lambda^{h}=$ hyperbolic geodesic in $\widetilde{M_{\mathrm{gf}}}$ joining $a, b$.

- $\lambda=\lambda^{a}=$ ambient quasigeodesic in $\widetilde{M_{0}}$ constructed from $\lambda^{h} \subset \widetilde{M_{\mathrm{gf}}}$.

- $\lambda_{\mathrm{cc}}=$ ambient quasigeodesic in $\widetilde{M_{\mathrm{cc}}}$ constructed from $\lambda \subset \widetilde{M_{0}}$.

- $\lambda^{c}=$ part of $\lambda_{\text {cc }}$ lying along $Z$-cusps or $Z+Z$-cusps.

- $\lambda^{b}=\lambda_{\mathrm{cc}}-\lambda^{c}$.

- $\mu_{i}=$ ambient geodesic in $\widetilde{S(i)}$.

- $\mu_{i}^{a}=$ ambient quasigeodesic in $\widetilde{S(i)}$ constructed from $\mu_{i}$.

- $\mu_{i}^{c}=$ part of $\mu_{i}^{a}$ lying along $Z$-cusps.

- $\mu_{i}^{b}=\mu_{i}^{a}-\mu_{i}^{c}$.

Recall that in the construction of $B_{1}(\lambda)$ from $B_{0}(\lambda)$ we construct $\mu$ from $\lambda$ by taking a $K$ (as in the discussion following Corollary 4.4) and choosing $p, q \in \widetilde{F} \cap N_{K}\left(B_{0}(\lambda)\right.$ ) with $d(p, q)$ maximal.

Now suppose $(M, P)$ is p-incompressible. (This assumption has effect from now till the end of Section 5.4, unless explicitly stated otherwise.) Then $\widetilde{F}$ is quasiconvex in the hyperbolic metric space $\widetilde{M_{0}}$. Hence, $\mu$ is a quasigeodesic in $\widetilde{M_{0}}$ and therefore lies in a $K_{1}$-neighborhood of $B_{0}(\lambda)$ (where $K_{1}$ depends on $K$ ). Further, since $\mu$ does not penetrate horoballs, the hyperbolic geodesic $\mu^{\prime}$ in $\widetilde{M_{0}}$ joining the end-points of $\mu$ 
can penetrate horoballs only for some bounded length $D_{1}$. Also, there is a subsegment $\lambda_{\mu}=\left[p^{\prime}, q^{\prime}\right] \subset \lambda$ such that $d\left(p, p^{\prime}\right) \leq K_{1}$ and $d\left(q, q^{\prime}\right) \leq K_{1}$. By Theorem 2.8, $\mu^{\prime}$ and $\lambda_{\mu}$ must have similar intersections with horoballs, ie there exists $C_{0}$ such that the following hold.

- If only one of $\mu^{\prime}$ and $\lambda_{\mu}$ penetrates or travels along the boundary of a horoball $\mathbf{H}$, then it can do so for a distance $\leq C_{0}$.

- If both $\mu^{\prime}$ and $\lambda_{\mu}$ enter (or leave) a horoball $\mathbf{H}$ then their entry (or exit) points are at a distance of at most $C_{0}$ from each other.

Then as in Corollary 5.2 if $x \in \mu^{b}$ there exists $C_{1}>0, x^{\prime \prime} \in \mu^{\prime}$ and $x^{\prime} \in \lambda^{b}$ such that

$$
\begin{gathered}
d\left(x, x^{\prime \prime}\right) \leq C_{1} \\
d\left(x^{\prime}, x^{\prime \prime}\right) \leq C_{1} .
\end{gathered}
$$

Hence, $d\left(x^{\prime}, x\right) \leq 2 C_{1}$. We have thus shown, (using Corollary 5.2) the following corollary.

Corollary 5.3 Suppose $(M, P)$ is a pared manifold with p-incompressible boundary. There exist $K, \epsilon>0$ such that for all $x \in \mu_{k}^{b} \subset B^{b}(\mu, \lambda)$ there exists a $(K, \epsilon)$ quasigeodesic ray $r_{x}:\{-1,0\} \cup \mathbb{N} \rightarrow B^{b}(\mu, \lambda)$ such that $r_{x}(k)=x, r_{x}(i) \in \mu_{i}^{b}$ for all $i=0,1,2, \ldots$ and $r_{x}(-1) \in \lambda^{b}$.

Note In the discussion preceding Corollary 5.3 above, all that we really required was that $\mu$ be a quasigeodesic in $\widetilde{M_{0}}$. Quasiconvexity of $\widetilde{F}$ (following from pincompressibility) ensured this. We therefore state the more general version below, as we shall require it to prove our main Theorem 5.12 where $\mathrm{p}$-incompressibility is relaxed to incompressibility.

Corollary 5.4 Suppose $(M, P)$ is a pared manifold with incompressible boundary. Given $D, \delta$ there exist $K, \epsilon$ such that the following holds: Suppose $\mu$ is a $(D, \delta)$ hyperbolic quasigeodesic in $\widetilde{M}_{0}$ lying on $\widetilde{F}$ for some $F$. Then for all $x \in \mu_{k}^{b} \subset$ $B^{b}(\mu, \lambda)$ there exists a $(K, \epsilon)$ quasigeodesic ray $r_{x}:\{-1,0\} \cup \mathbb{N} \rightarrow B^{b}(\mu, \lambda)$ such that $r_{x}(k)=x, r_{x}(i) \in \mu_{i}^{b}$ for all $i=0,1,2, \ldots$ and $r_{x}(-1) \in \lambda^{b}$.

The hypothesis of Corollary 5.4 is satisfied if $\mu$ moves along boundaries of horoballs for uniformly bounded distances. Equivalently, this is satisfied if the hyperbolic geodesic $\mu^{h}$ joining the end-points of $\mu$ penetrates horoballs by a uniformly bounded amount. Actually, we do not need even this much. $\mu^{h}$ should penetrate exceptional horoballs (see definition below) by a bounded amount. 
Definition A cusp of $M_{\mathrm{gf}}$ is said to be exceptional if there exist closed curves carried by the cusp (ie lying on its boundary horocycle or horosphere) which are homotopic to non-peripheral curves on some other boundary component of $M_{\mathrm{gf}}$. Exceptional horoballs are lifts of exceptional cusps. A geodesic is said to penetrate a horoball $H$ by at most $D$ if any subsegment of it lying inside $H$ has length less than or equal to D.

The sufficient condition for the hypothesis of Corollary 5.4 to hold is set forth in the following lemma.

Lemma 5.5 Let $F$ be some boundary component of $M_{0}$ and $\mu \subset \widetilde{F}$ be a geodesic segment in the path metric on $\widetilde{F}$. Let $\mu^{h}$ be the hyperbolic geodesic in $\widetilde{M_{\mathrm{gf}}}$ joining the end-points of $\mu$. Given $D>0$ there exist $K, \epsilon$ such that if $\mu^{h}$ penetrates exceptional horoballs by at most $D$ then $\mu$ is a $(K, \epsilon)$ quasigeodesic in $\widetilde{M_{0}}$.

\subsection{Ambient and hyperbolic geodesics}

Recall

- $\lambda^{h}=$ hyperbolic geodesic in $\widetilde{M_{\mathrm{gf}}}$ joining $a, b$,

- $\lambda=\lambda^{a}=$ ambient quasigeodesic in $\widetilde{M_{0}}$ constructed from $\lambda^{h} \subset \widetilde{M_{\mathrm{gf}}}$,

- $\beta^{h}=$ geodesic in $\widetilde{N^{h}}$ joining $a, b$,

- $\beta_{\mathrm{amb}}^{0}=$ ambient quasigeodesic in $\widetilde{N_{0}}$ obtained from $\beta^{h}$ by replacement of hyperbolic by 'Euclidean' geodesic segments for $Z$-horoballs in $\widetilde{N^{h}}$,

- $\beta_{\mathrm{amb}}=\Pi_{\lambda}\left(\beta_{\mathrm{amb}}^{0}\right)$.

By construction, the hyperbolic geodesic $\beta^{h}$ and the ambient quasigeodesic $\beta_{\mathrm{amb}}^{0}$ agree exactly off horoballs. $\beta_{\mathrm{amb}}$ is constructed from $\beta_{\mathrm{amb}}^{0}$ by projecting it onto $B_{\lambda}$ and so by Theorem 4.20, it is an ambient quasigeodesic. But it might 'backtrack'. Hence, we shall modify it such that it satisfies the no backtracking condition. First, observe by Theorem 2.8 that $\beta^{h}, \beta_{\mathrm{amb}}^{0}$ track each other off some $K$-neighborhood of horoballs.

The advantage of working with $\beta_{\mathrm{amb}}$ is that it lies on $B_{\lambda}$. However, it might backtrack. Now, recall that $B^{a}(\lambda)$ was constructed from $B_{\lambda}$ by replacing each $B(\mu)$ by $B^{a}(\mu)$. We can therefore choose an ambient quasigeodesic lying on $B^{a}(\lambda)$ that tracks $\beta_{\text {amb }}$ throughout its length. The advantage of switching to $B^{a}(\lambda)$ is that it is constructed from ambient geodesics without backtracking lying on the universal covers of equispaced pleated surfaces. We shall (abusing notation) call this new ambient quasigeodesic $\beta_{\mathrm{amb}}$. Thus $\beta_{\text {amb }}$ now lies on $B^{a}(\lambda)$ rather than $B_{\lambda}$. 
Lemma 5.6 There exists $C>0$ such that for all $x \in \mu_{i}^{b} \subset B^{a}(\mu, \lambda) \subset B^{a}(\lambda)$ if $\lambda^{h}$ lies outside $B_{n}(p)$ for a fixed reference point $p \in \widetilde{M^{h}}$, then $x$ lies outside an $\frac{n-C}{C+1}$ ball about $p$ in $\widetilde{N_{0}}$.

Proof By Corollary 5.3, $r_{x}(-1) \in \lambda^{b}$. Since $\lambda^{b}$ is a part of $\lambda^{h}$, therefore $r_{x}(-1)$ lies outside $B_{n}(p)$. By Corollary 5.3, there exists $C>0$ such that for all $i, j \in$ $\{-1,0,1,2, \ldots\}$,

$$
|i-j| \leq d\left(r_{x}(i), r_{x}(j)\right) \leq C|i-j|
$$

Also, $d(x, p) \geq i$ since $x \in \mu_{i}^{b}$. (Here distances are all measured in $\widetilde{N_{0}}$.) Hence,

$$
\begin{aligned}
d(x, p) & \geq \max \{i, n-C(i+1)\} \\
& \geq \frac{n-C}{C+1}
\end{aligned}
$$

This proves the result.

If $x \in B^{a}(\lambda)$, then $x \in \lambda^{b}$ or $x \in \lambda^{c}$ or $x \in B^{b}(\mu)$ or $x \in B^{c}(\mu)$ for some $\mu$. Hence $x \in B^{a}(\lambda)$ implies that either $x$ lies on some horosphere bounding some $\mathbf{H} \in \mathcal{H}$ or, from Lemma 5.6 above, $d(x, p) \geq \frac{n-C}{C+1}$. Since $\beta_{\text {amb }}$ lies on $B^{a}(\lambda)$, we conclude that $\beta_{\text {amb }}$ is an ambient quasigeodesic in $N_{0}$ such that every point $x$ on $\beta_{\text {amb }}$ either lies on some horosphere bounding some $\mathbf{H} \in \mathcal{H}$ or, from Lemma 5.6 above, $d(x, p) \geq \frac{n-C}{C+1}$.

McMullen [26] shows (cf Theorem 2.7) that in $\widetilde{N^{h}}$, any such ambient quasigeodesic $\beta_{\text {amb }}$ lies in a bounded neighborhood of $\beta^{h} \cup \mathcal{H}\left(\beta^{h}\right)$. We do not as yet know that $\beta_{\text {amb }}$ does not backtrack, but we can convert it into one without much effort. (Note that Theorem 2.7 does not require "no backtracking".) Let $\Pi$ denote nearest point projection of $\widetilde{N^{h}}$ onto $\lambda^{h} \cup \mathcal{H}$. Since $\Pi$ is a large-scale Lipschitz retract (Theorem 4.20), $\Pi\left(\beta_{\mathrm{amb}}\right)=\beta_{1}$ is again an ambient quasigeodesic in $\widetilde{N_{0}}$. Further, $\beta_{1}$ tracks $\beta_{\text {amb }}$ throughout its length as $\Pi$ moves points on $\beta_{\text {amb }}$ through a uniformly bounded distance (Theorem 2.7). Now $\beta_{1}$ might backtrack, but it can do so in a trivial way, ie if $\beta_{1}$ re-enters a horoball after leaving it, it must do so at exactly the point where it leaves it. Removing these 'trivial backtracks', we obtain an ambient quasigeodesic without backtracking $\beta$ which tracks $\beta_{\mathrm{amb}}$ throughout its length.

Note On the one hand $\beta$ is an ambient quasigeodesic without backtracking. Hence, it reflects the intersection pattern of $\beta^{h}$ with horoballs. On the other hand, it tracks $\beta_{\mathrm{amb}}$ whose properties we already know from Corollary 5.3 above. 
Since, of $\beta$ and $\beta^{h}$, one is an ambient quasigeodesic without backtracking, and the other a hyperbolic geodesic joining the same pair of points, we conclude from Theorem 2.8 that they have similar intersection patterns with horoballs, ie there exists $C_{0}$ such that

- if only one of $\beta$ and $\beta^{h}$ penetrates or travels along the boundary of a horoball $\mathbf{H}$, then it can do so for a distance $\leq C_{0}$,

- if both $\beta$ and $\beta^{h}$ enter (or leave) a horoball $\mathbf{H}$ then their entry (or exit) points are at a distance of at most $C_{0}$ from each other.

Again, since $\beta$ tracks $\beta_{\mathrm{amb}}$, we conclude that there exists $C>0$ such that $\beta$ lies in a $C$-neighborhood of $\beta_{\mathrm{amb}}$ and hence from Lemma 5.6,

- every point $x$ on $\beta$ either lies on some horosphere bounding some $\mathbf{H} \in \mathcal{H}$ or, $d(x, p) \geq \frac{n-C}{C+1}-C$.

The above three conditions on $\beta$ and $\beta^{h}$ allow us to deduce the following (identical to the third) condition for $\beta^{h}$ in the $\mathrm{p}$-incompressible case.

Proposition 5.7 Suppose $(M, P)$ has p-incompressible boundary. Then every point $x$ on $\beta^{h}$ either lies inside some horoball $\mathbf{H} \in \mathcal{H}$ or, $d(x, p) \geq \frac{n-C}{C+1}-C=m(n)$.

We have denoted $\frac{n-C}{C+1}-C$ by $m(n)$, so that $m(n) \rightarrow \infty$ as $n \rightarrow \infty$. The above Proposition asserts that the geodesic $\beta^{h}$ lies outside large balls about $p$ modulo horoballs. By Lemma 2.4 this is almost enough to guarantee the existence of a CannonThurston map.

Again as in Corollary 5.4 it is not necessary to restrict ourselves to the p-incompressible case. We deduce, using Corollary 5.4 and Lemma 5.5 the following proposition.

Proposition 5.8 Suppose $(M, P)$ has incompressible boundary $\partial_{0} P$. Given $D, n$ there exist $m(n, D)$ such that the following holds: Let $\lambda^{h}, \beta^{h}, \lambda$ and $\mu$ be as before. If each $\mu$ penetrates exceptional horoballs by at most $D$, then every point $x$ on $\beta^{h}$ either lies inside some horoball $\mathbf{H} \in \mathcal{H}$ or, $d(x, p) \geq m(n, D)$, for some function $m(n, D)$ where $m(n, D) \rightarrow \infty$ as $n \rightarrow \infty$ for each fixed $D$.

We can divide $\beta^{h}$ into two subsets $\beta^{c}$ and $\beta^{b}$ as earlier. $\beta^{c}=\beta^{h} \cap \mathcal{H}$ is the intersection of $\beta^{h}$ with horoballs, and $\beta^{b}=\beta^{h}-\beta^{c}$. The main theorem of this paper under the assumption of $p$-incompressibility follows. Recall that $M_{\mathrm{gf}}$ is identified with its homeomorphic image in $N^{h}$ taking cusps to cusps. 
Theorem 5.9 Suppose that $N^{h} \in H(M, P)$ is a hyperbolic structure of bounded geometry on a pared manifold $(M, P)$ with p-incompressible boundary. Let $M_{\mathrm{gf}}$ denotes a geometrically finite hyperbolic structure adapted to $(M, P)$, then the map $i: \widetilde{M_{\mathrm{gf}}} \rightarrow \widetilde{N^{h}}$ extends continuously to the boundary $\widehat{i}: \widehat{M_{\mathrm{gf}}} \rightarrow \widehat{N^{h}}$. If $\Lambda$ denotes the limit set of $\widetilde{M}$, then $\Lambda$ is locally connected.

Proof Let $\lambda^{h}$ be a geodesic segment in $\widetilde{M_{\mathrm{gf}}}$ lying outside $N_{n}(p)$ for some fixed reference point $p$. Fix neighborhoods of the cusps and lift them to the universal cover. Let $\mathcal{H}$ denote the set of horoballs. Assume without loss of generality that $p$ lies outside horoballs. Let $\beta^{h}$ be the hyperbolic geodesic in $\widetilde{N^{h}}$ joining the endpoints of $\lambda^{h}$. Further, let $\beta^{h}=\beta^{b} \cup \beta^{c}$ as above. Then by Proposition 5.7, $\beta^{b}$ lies outside an $m(n)$ ball about $p$, with $m(n) \rightarrow \infty$ as $n \rightarrow \infty$.

Next, let $\mathbf{H}_{1}$ be some horoball that $\beta^{h}$ meets. Then the entry and exit points $u$ and $v$ of $\beta^{h}$ into and out of $\mathbf{H}_{1}$ lie outside an $m(n)$ ball about $p$. Let $z$ be the point on the boundary sphere that $\mathbf{H}_{1}$ is based at. Then for any sequence $x_{i} \in \mathbf{H}_{1}$ with $d\left(p, x_{i}\right) \rightarrow \infty, x_{i} \rightarrow z$. If $\left\{x_{i}\right\}$ and $\left\{y_{i}\right\}$ denote two such sequences, then the visual diameter of the set $\left\{x_{i}, y_{i}\right\}$ must go to zero. Hence, if $\left[x_{i}, y_{i}\right]$ denotes the geodesic joining $x_{i}, y_{i}$ then $d\left(p,\left[x_{i}, y_{i}\right]\right) \rightarrow \infty$. Since, $u, v$ lie outside an $m(n)$ ball, there exists some function $\psi$, such that the geodesic $[u, v]$ lies outside a $\psi(m(n))$ ball around $p$, where $\psi(k) \rightarrow \infty$ as $k \rightarrow \infty$.

We still need to argue that the function $\psi$ is independent of the horoball $\mathbf{H}_{1}$. Assume that the lifts of horoballs miss the base-point $p$. If the conclusion fails, there exist a sequence of horoballs $\mathbf{H}_{i}$, points $x_{i}, y_{i}$ on the boundary of $\mathbf{H}_{i}$, such that $\left[x_{i}, y_{i}\right] \subset \mathbf{H}_{i}$ passes through some fixed $N$-ball $B_{N}(p)$ about $p$. Passing to a subsequence and extracting a limit we may assume that the horoballs $\mathbf{H}_{i}$ converge to some horoball $\mathbf{H}$ cutting $B_{N}(p)$ (possibly touching $p$ ). Then the above discussion for $\mathbf{H}_{1}$ furnishes the required contradiction. Hence, the choice of this function $\psi$ does not depend on $\mathbf{H}_{1}$. We conclude that there exists such a function for all of $\beta^{c}$. We have thus established:

- $\beta^{b}$ lies outside an $m(n)$ ball about $p$,

- $\beta^{c}$ lies outside a $\psi(m(n))$ ball about $p$,

- $m(n)$ and $\psi(m(n))$ tend to infinity as $n \rightarrow \infty$.

Define $f(n)=\min (m(n), \psi(m(n)))$. Then $\beta^{h}$ lies outside an $f(n)$ ball about $p$ and $f(n) \rightarrow \infty$ as $n \rightarrow \infty$.

By Lemma $2.4 i: \widetilde{M_{\mathrm{gf}}} \rightarrow \widetilde{N^{h}}$ extends continuously to the boundary $\widehat{i}: \widehat{M_{\mathrm{gf}}} \rightarrow \widehat{N^{h}}$ This proves the first statement of the theorem. 
Now, for any geometrically finite Kleinian group, its limit set is locally connected.(See, for instance, Anderson-Maskit [2].) Hence, the limit set of $\widetilde{M_{\mathrm{gf}}}$ is locally connected. Further, the continuous image of a compact locally connected set is locally connected Hocking-Young [22]. Hence, if $\Lambda$ denotes the limit set of $\widetilde{N^{h}}$, then $\Lambda$ is locally connected. This proves the theorem.

Again as in Corollary 5.4 and Proposition 5.8 it is not necessary to restrict ourselves to the p-incompressible case. In Theorem 5.9 above we removed the clause on being within horoballs from Proposition 5.7. In the proof of Theorem 5.9 above the one new thing we had shown was that geodesics entering and leaving horoballs at a large distance from the reference point $p$, itself lies at a large distance from $p$. Using this observation along with Proposition 5.8 we easily deduce the following corollary.

Corollary 5.10 Suppose $(M, P)$ has incompressible boundary $\partial_{0} P$. Given $D, n$ there exist $m(n, D)$ such that the following holds: Let $\lambda^{h}, \beta^{h}$ be as before. If $\lambda^{h}$ penetrates exceptional horoballs by at most $D$ and if $\lambda^{h}$ lies outside $B_{n}(p)$ in $\widetilde{M_{\mathrm{gf}}}$ then $\beta^{h}$ lies outside a ball of radius $m(n, D)$, for some function $m(n, D)$ where $m(n, D) \rightarrow \infty$ as $n \rightarrow \infty$ for each fixed $D$.

The above proposition will be useful in the next subsection when we go from $p-$ incompressibility to incompressibility.

\subsection{From p-incompressibility to incompressibility}

In this subsection we shall use Corollary 5.10 to relax the hypothesis of p-incompressibility. Recall that we started with a hyperbolic geodesic $\lambda^{h}$ in $\widetilde{M_{\mathrm{gf}}}$ and then modified it along horoballs to obtain $\lambda$. In this subsection we first consider subsegments $\lambda_{1}^{h}, \ldots, \lambda_{k}^{h}$ where each $\lambda_{i}^{h}$ starts and ends on horospheres bounding horoballs, and the complementary segments (of $\lambda^{h}$ ) lie inside horoballs. This decomposition is made in such a way that the starting and ending points of $\lambda_{i}^{h}$ lie on exceptional horoballs (see previous subsection for definitions.) Having completed this decomposition we consider hyperbolic geodesics $\beta_{i}^{h}$ in $\widetilde{N^{h}}$ joining the end-points of $\lambda_{i}^{h}$. If we ensure that the geodesics $\lambda_{i}^{h}$ penetrate exceptional horoballs by a uniformly bounded amount, then by Corollary 5.10 above, we can ensure that the segments $\beta_{i}^{h}$ lie outside a large ball about $p$.

If further, we can ensure that

- the $\beta_{i}^{h}$ penetrate the horoballs they start and end on by a bounded amount, 
- and that the terminal point of $\beta_{i}^{h}$ and the initial point of $\beta_{i+1}^{h}$ are separated by more than a critical amount,

then (by appealing to the fact that local quasigeodesics are global quasigeodesics $[20 ; 19])$ we conclude that the union of the $\beta_{i}^{h}$ with the hyperbolic geodesics interpolating between them (and lying entirely within horoballs) is a (uniform) hyperbolic quasigeodesic.

Since each $\beta_{i}^{h}$ lies outside a large ball about $p$, it follows that their union along with interpolating geodesics does so too. This union being a quasigeodesic, the geodesic $\beta^{h}$ joining the end-points of $\lambda$ must also lie outside a large ball about $p$. By Lemma 2.4 the existence of a Cannon-Thurston map follows.

We now furnish the details of the above argument.

First note from Corollary 5.10 that given $D$ there exists $m(n, D)$ such that if $\lambda_{1}^{h}$ be a subsegment of $\lambda^{h}$ penetrating exceptional horoballs by at most $D$, and if $\lambda^{h}$ (and hence $\lambda_{1}^{h}$ ) lies outside $B_{n}(p)$ then $\beta_{1}^{h}$ (the hyperbolic geodesic in $\widetilde{N^{h}}$ joining the endpoints of $\left.\lambda_{1}^{h}\right)$ lies outside an $m(n, D)$-ball about $p$.

The next proposition follows from the fact that local quasigeodesics are global quasigeodesics in hyperbolic space. (See Gromov [20, Proposition 7.2C] and CoornaertDelzant-Papadopoulos [16].)

Proposition 5.11 There exist $D, K, \epsilon$ such that the following holds.

Suppose $\beta^{x}$ is a path in $\widetilde{N^{h}}$ such that $\beta^{x}$ can be decomposed into finitely many geodesic segments $\beta_{1}, \ldots, \beta_{k}$. Further suppose that the starting and ending point of each $\beta_{i}$ lie on exceptional horospheres (except possibly the starting point of $\beta_{1}$ and the ending-point of $\beta_{k}$ ) meeting the horospheres at right angles. Also suppose that the 'even segments' $\beta_{2 i}$ lie entirely within exceptional horoballs and have length greater than $D$. Then $\beta^{x}$ is a $(K, \epsilon)$ quasigeodesic.

Proof Idea Since geodesic segments lying outside horoballs meet horospheres at right angles, successive pieces meet at an angle that is bounded below. Hence, if $x_{i}, z_{i} \in \beta_{i}, \beta i+1$ respectively and $\beta_{i} \cap \beta i+1=y_{i}$, then $\left[x_{i}, y_{i}\right] \cup\left[y_{i}, z_{i}\right]$ is a (uniform) $\left(K_{1}, \epsilon_{1}\right)$-quasigeodesic of length $\geq D+1$ assuming that any two horoballs are separated by a distance of at least one. That is every segment of length $D+1$ is a $\left(K_{1}, \epsilon_{1}\right)$-quasigeodesic, ie see [16, Chapter 3]) it is a local quasigeodesic. If $D$ is sufficiently long, stability of quasigeodesics (Theorems 1.2, 1.3, 1.4 of [16, Chapter 3]) ensures that $\beta^{x}$ is a $(K, \epsilon)$ quasigeodesic for some $K, \epsilon$ independent of $\beta$. 
In fact we do not need that the horoballs be exceptional, but that they be uniformly separated, ie the distance between any two horospheres is uniformly bounded below. This is clearly true for the exceptional horoballs since they are lifts of certain given cusps in $M_{\mathrm{gf}}$ which in turn are finitely many in number and have been chosen uniformly separated.

Next, we need to look closely at how we use Corollary 5.3 to prove Theorem 5.9. Note that $\beta_{\mathrm{amb}}$ is an ambient quasigeodesic independent of the hypothesis of $p-$ incompressibility. It is in concluding that $\beta_{\mathrm{amb}}$ lies outside a large $m(n)$-ball about the reference point $p$ that we needed to construct quasigeodesic rays going down to $\lambda$.

Now let $\lambda^{h}=[a, x]$ be a hyperbolic geodesic in $\widetilde{M_{\mathrm{gf}}}$ having the end-point $x$ on an exceptional horosphere bounding a horoball $H$. By our construction of $B_{\lambda}$ we note that $B_{\lambda}$ is quasi-isometrically embedded in $\widetilde{N}$ and further that $\beta_{\text {amb }}$ (the ambient quasigeodesic corresponding to $\lambda^{h}$ ) meets $H$ either at $x$ (if $H$ is a $(Z+Z$ )-horoball) or at some point on $r_{x}$ (for a quasigeodesic ray $r_{x}$ constructed through $x$ and lying in the universal cover $\widetilde{E}$ of an end $E$ of the manifold) if $H$ be a $Z$-horoball.

Now suppose $\lambda_{1}^{h}=[a, x], \lambda_{2}^{h}=[x, y]$ and $\lambda_{3}^{h}=[y, b]$ be three hyperbolic geodesic segments such that $[x, y] \subset \mathbf{H}$ and $\lambda_{3}^{h}$ meets $H$ at $y$. Again, as before the corresponding ambient quasigeodesic $\beta_{\mathrm{amb}}^{1}$ meets $\mathbf{H}$ at $x$ or at some point along an $r_{x}$ and $\beta_{\mathrm{amb}}^{3}$ meets $\mathbf{H}$ at $y$ or at some point along an $r_{y}$.

In any case, the distance between the entry point of $\beta_{1}^{h}$ (the hyperbolic geodesic joining the end-points of $\beta_{\mathrm{amb}}^{1}$ ) into $H$ and the exit point of $\beta_{3}^{h}$ (the hyperbolic geodesic joining the end-points of $\beta_{\mathrm{amb}}^{3}$ ) from $H$ is greater than $d(x, y)-2 C_{0}$ for some $C_{0}$ depending on the quasiconvexity constant of $\beta_{\mathrm{amb}}^{i}\left(\mathrm{i}=1,3\right.$ ) by Theorem 2.8. Thus $C_{0}$ depends only on the quasi-Lipschitz constant of $\Pi_{\lambda}$.

We are now in a position to break $\lambda^{h}$ into pieces. Let $\lambda_{2 i}^{h}$ denote the maximal subsegments of $\lambda^{h}$ lying inside exceptional horoballs and having length greater than $\left(2 C_{0}+D_{0}\right)$. Let $\lambda_{2 i-1}^{h}$ denote the complementary segments. Now, let $\overline{\beta_{2 i-1}^{h}}$ be the hyperbolic geodesic in $\widetilde{N^{h}}$ joining the endpoints of $\lambda_{2 i-1}^{h}$. Then the entry point of $\overline{\beta_{2 i-1}^{h}}$ into the exceptional horoball $\mathbf{H}$ it terminates on lies at a distance greater than $\left(2 C_{0}+D_{0}-2 C_{0}\right)=D_{0}$ from the exit point of $\overline{\beta_{2 i+1}^{h}}$ from the same horoball $\mathbf{H}$. Shorten the hyperbolic geodesic $\overline{\beta_{2 i-1}^{h}}$ if necessary by cutting it off at the entry point into $\mathbf{H}$. Let $\beta_{2 i-1}^{h}$ denote the resultant geodesic. By Corollary $5.10 \beta_{2 i-1}^{h}$ being a subsegment of $\overline{\beta_{2 i-1}^{h}}$ lies at a distance of at least $m\left(n,\left(2 C_{0}+D_{0}\right)\right)=m_{1}(n)$ from the reference point $p$. 
Next denote by $\beta_{2 i}^{h}$ the hyperbolic geodesic lying entirely within $\mathbf{H}$ joining the entry point of $\beta_{2 i-1}^{h}$ into $\mathbf{H}$ to the exit point of $\beta_{2 i+1}^{h}$ from $\mathbf{H}$. The initial and terminal points of $\beta_{2 i}^{h}$ lying on $\mathbf{H}$ are at a distance of at least $m_{1}(n)$ from $p$. Therefore each $\beta_{2 i}^{h}$ and hence the union of all the $\beta_{i}^{h}$ lie outside an $m_{2}(n)$ ball about $p$ where $m_{2}(n) \rightarrow \infty$ as $n \rightarrow \infty$. Further, the union of the segments $\beta_{i}^{h}$ is a hyperbolic quasigeodesic by Proposition 5.11 and hence lies at a bounded distance $D^{\prime}$ from the hyperbolic geodesic $\beta^{h}$ joining the endpoints of $\lambda$ (ie the end-points of $\lambda^{h}$ ). Let $m_{3}(n)=m_{2}(n)-D^{\prime}$. Then what we have shown amounts to the following.

- If $\lambda^{h}$ lies outside $B_{n}(p)$, then $\beta^{h}$ lies outside a ball of radius $m_{3}(n)$ about $p$.

- $m_{3}(n) \rightarrow \infty$ as $n \rightarrow \infty$.

Coupled with Lemma 2.4 this proves the main theorem of this paper.

Theorem 5.12 Suppose that $N^{h} \in H(M, P)$ is a hyperbolic structure of bounded geometry on a pared manifold $(M, P)$ with incompressible boundary $\partial_{0} M$. Let $M_{\mathrm{gf}}$ denotes a geometrically finite hyperbolic structure adapted to $(M, P)$. Then the map $i: \widetilde{M_{\mathrm{gf}}} \rightarrow \widetilde{N^{h}}$ extends continuously to the boundary $\hat{i}: \widehat{M_{\mathrm{gf}}} \rightarrow \widehat{N^{h}}$. If $\Lambda$ denotes the limit set of $\widetilde{M}$, then $\Lambda$ is locally connected.

\section{Examples and consequences}

As mentioned in the Introduction, the simplest non-trivial examples to which Theorem 5.12 applies are hyperbolic three manifolds of finite volume fibering over the circle. These include the original examples of Cannon and Thurston [15] as well as those of Bowditch [11; 12].

The next set of examples are those three manifolds homeomorphic to the product of a surface and $\mathbb{R}$. These were dealt with in Minsky's work [28] and the punctured surface case was dealt with by Bowditch $[11 ; 12]$.

The case of three manifolds of freely indecomposable fundamental group were dealt with independently by Klarreich [23] and the author [36]. In fact, Klarreich's theorem is really a reduction theorem which effectively says that if one can prove CannonThurston for closed surface groups (of some given geometry) then one can also prove it for 3 manifolds whose ends have the same geometry. In combination with Minsky's adaptation of the original proof of Cannon-Thurston, this proves the theorem for bounded geometry 3-manifolds with incompressible (closed surface) boundary. In [36] we had approached this problem directly and had given different proofs of these results. In this paper we have continued the approach in [36] to prove the analogue of the above results in the presence of parabolics. 
Incompressibility and compressibility One problem that has not been addressed by this paper or its predecessor [36] is the case of compressible $\partial_{0} M$. Miyachi [37] (see also Souto [45]) has solved this problem in the bounded geometry case, when there are no cusps. In [40] we shall combine the reduction techniques of this paper with a coarse version of Miyachi's argument to settle affirmatively the question of existence of Cannon-Thurston maps for finitely generated Kleinian groups.

Coarse framework Many of the arguments of this paper rightfully belong to the domain of 'coarse' or 'asymptotic' or 'large-scale' geometry in spirit and it is more than likely that they may be generalized to this setting. In [35] and [36], we had proven the following theorems.

Theorem 6.1 [35] Let $G$ be a hyperbolic group and let $H$ be a hyperbolic subgroup that is normal in $G$. Let $i$ : $\Gamma_{H} \rightarrow \Gamma_{G}$ be the continuous proper embedding of $\Gamma_{H}$ in $\Gamma_{G}$ described above. Then $i$ extends to a continuous map $\hat{i}$ from $\widehat{\Gamma_{H}}$ to $\widehat{\Gamma_{G}}$.

Theorem 6.2 [36] Let $(X, d)$ be a tree $(T)$ of hyperbolic metric spaces satisfying the qi-embedded condition. Let $v$ be a vertex of $T$. If $X$ is hyperbolic $i_{v}: X_{v} \rightarrow X$ extends continuously to $\widehat{i_{v}}: \widehat{X_{v}} \rightarrow \widehat{X}$.

In this paper we have described a fairly general way of handling cusps. The generalization to 'coarse geometry' involves dealing with relatively hyperbolic groups a la Gromov [20], Farb [17] and Bowditch [8].

In [41], the author and Abhijit Pal generalize Theorem 6.2 to the context of trees of (strongly) relatively hyperbolic metric spaces. In [43], Pal adapts the techniques of this paper and generalizes Theorem 6.1 to (strongly) relatively hyperbolic normal subgroups of (strongly) relatively hyperbolic groups.

\section{References}

[1] W Abikoff, Kleinian groups-geometrically finite and geometrically perverse, from: "Geometry of group representations (Boulder, CO, 1987)", Contemp. Math. 74, Amer. Math. Soc., Providence, RI (1988) 1-50 MR957510

[2] J W Anderson, B Maskit, On the local connectivity of limit set of Kleinian groups, Complex Variables Theory Appl. 31 (1996) 177-183 MR1423249

[3] M Bestvina, Geometric group theory problem list (2004) Available at http:// math.utah.edu/ bestvina

[4] M Bestvina, M Feighn, A combination theorem for negatively curved groups, J. Differential Geom. 35 (1992) 85-101 MR1152226 
[5] M Bestvina, M Feighn, M Handel, Laminations, trees, and irreducible automorphisms of free groups, Geom. Funct. Anal. 7 (1997) 215-244 MR1445386

[6] M Bestvina, M Handel, Train tracks and automorphisms of free groups, Ann. of Math. (2) 135 (1992) 1-51 MR1147956

[7] F Bonahon, Bouts des variétés hyperboliques de dimension 3, Ann. of Math. (2) 124 (1986) 71-158 MR847953

[8] B H Bowditch, Relatively hyperbolic groups, preprint, Southampton (1997)

[9] B H Bowditch, A topological characterisation of hyperbolic groups, J. Amer. Math. Soc. 11 (1998) 643-667 MR1602069

[10] B H Bowditch, Convergence groups and configuration spaces, from: "Geometric group theory down under (Canberra, 1996)", (J Cossey, C F Miller, W D Neumann, M Shapiro, editors), de Gruyter, Berlin (1999) 23-54 MR1714838

[11] B H Bowditch, Stacks of hyperbolic spaces and ends of 3 manifolds, preprint, Southampton (2002)

[12] B H Bowditch, The Cannon-Thurston map for punctured-surface groups, Math. Z. 255 (2007) 35-76 MR2262721

[13] J F Brock, Iteration of mapping classes and limits of hyperbolic 3-manifolds, Invent. Math. 143 (2001) 523-570 MR1817644

[14] J F Brock, R D Canary, Y N Minsky, The Classification of Kleinian surface groups II: The Ending Lamination Conjecture, preprint (2004)

[15] J W Cannon, W P Thurston, Group invariant Peano curves, Geom. Topol. 11 (2007) 1315-1355 MR2326947

[16] M Coornaert, T Delzant, A Papadopoulos, Géométrie et théorie des groupes, Lecture Notes in Mathematics 1441, Springer, Berlin (1990) MR1075994 Les groupes hyperboliques de Gromov. [Gromov hyperbolic groups], With an English summary

[17] B Farb, Relatively hyperbolic groups, Geom. Funct. Anal. 8 (1998) 810-840 MR1650094

[18] W J Floyd, Group completions and limit sets of Kleinian groups, Invent. Math. 57 (1980) 205-218 MR568933

[19] É Ghys, P de la Harpe (editors), Sur les groupes hyperboliques d'après Mikhael Gromov, Progress in Mathematics 83, Birkhäuser, Boston (1990) MR1086648 Papers from the Swiss Seminar on Hyperbolic Groups held in Bern, 1988

[20] M Gromov, Hyperbolic groups, from: "Essays in group theory", Math. Sci. Res. Inst. Publ. 8, Springer, New York (1987) 75-263 MR919829

[21] M Gromov, Metric structures for Riemannian and non-Riemannian spaces, Progress in Mathematics 152, Birkhäuser, Boston (1999) MR1699320 Based on the 1981 French original 
[22] J G Hocking, G S Young, Topology, Addison-Wesley Publishing Co., Reading, MALondon (1961) MR0125557

[23] E Klarreich, Semiconjugacies between Kleinian group actions on the Riemann sphere, Amer. J. Math. 121 (1999) 1031-1078 MR1713300

[24] C T McMullen, Amenability, Poincaré series and quasiconformal maps, Invent. Math. 97 (1989) 95-127 MR999314

[25] C T McMullen, Iteration on Teichmüller space, Invent. Math. 99 (1990) 425-454 MR1031909

[26] C T McMullen, Local connectivity, Kleinian groups and geodesics on the blowup of the torus, Invent. Math. 146 (2001) 35-91 MR1859018

[27] Y N Minsky, Teichmüller geodesics and ends of hyperbolic 3-manifolds, Topology 32 (1993) 625-647 MR1231968

[28] Y N Minsky, On rigidity, limit sets, and end invariants of hyperbolic 3-manifolds, J. Amer. Math. Soc. 7 (1994) 539-588 MR1257060

[29] Y N Minsky, The classification of punctured-torus groups, Ann. of Math. (2) 149 (1999) 559-626 MR1689341

[30] Y N Minsky, Bounded geometry for Kleinian groups, Invent. Math. 146 (2001) 143-192 MR1859020

[31] Y N Minsky, The classification of Kleinian surface groups I: Models and Bounds, preprint (2002)

[32] Y N Minsky, End invariants and the classification of hyperbolic 3-manifolds, from: "Current developments in mathematics, 2002", Int. Press, Somerville, MA (2003) 181217 MR2062319

[33] M Mitra, PhD thesis, UC Berkeley (1997)

[34] M Mitra, Ending laminations for hyperbolic group extensions, Geom. Funct. Anal. 7 (1997) 379-402 MR1445392

[35] M Mitra, Cannon-Thurston maps for hyperbolic group extensions, Topology 37 (1998) 527-538 MR1604882

[36] M Mitra, Cannon-Thurston maps for trees of hyperbolic metric spaces, J. Differential Geom. 48 (1998) 135-164 MR1622603

[37] H Miyachi, Semiconjugacies between actions of topologically tame Kleinian groups, preprint (2002)

[38] M Mj, Cannon-Thurston Maps for Surface Groups I: Amalgamation Geometry and Split Geometry (2005) arXiv:math.GT/0512539

[39] M Mj, Cannon-Thurston maps, $i$-bounded geometry and a theorem of McMullen (2005) arXiv:math.GT/0511041 
[40] M Mj, Cannon-Thurston Maps for Surface Groups II: Split Geometry and the Minsky Model (2006) arXiv:math.GT0607509

[41] M Mj, A Pal, Relative Hyperbolicity, Trees of Spaces and Cannon-Thurston Maps (2007) arXiv:0708.3578

[42] R M Myers, From Beowulf to Virginia Woolf: An astounding and wholly unauthorized history of English literature (1952)

[43] A Pal, Relative Hyperbolic Extensions of Groups and Cannon-Thurston Maps (2008) arXiv:0801.0933

[44] G P Scott, Compact submanifolds of 3-manifolds, J. London Math. Soc. (2) 7 (1973) 246-250 MR0326737

[45] J Souto, Cannon-Thurston maps for thick free groups, preprint (2006)

[46] W P Thurston, The geometry and topology of 3-manifolds, Princeton University notes (1980)

[47] W P Thurston, Hyperbolic structures on 3-manifolds. I. Deformation of acylindrical manifolds, Ann. of Math. (2) 124 (1986) 203-246 MR855294

[48] W P Thurston, Hyperbolic structures on 3-manifolds. III. Deformation of 3-manifolds with incompressible boundary, preprint (1986)

[49] A Yaman, A topological characterisation of relatively hyperbolic groups, J. Reine Angew. Math. 566 (2004) 41-89 MR2039323

School of Mathematical Sciences, RKM Vivekananda University PO Belur Math, Dt Howrah, WB-711202, India

mahan.mj@gmail.com

http://people.rkmvu.ac.in/ mahan/

Proposed: Jean-Pierre Otal

Seconded: Benson Farb, Dave Gabai
Received: 17 February 2006 Revised: 18 September 2008 